\title{
基于 $\left[\mathrm{Mo}(\mathrm{CN})_{7}\right]^{4-}$ 的分子磁性材料研究进展
}

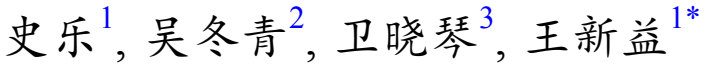

1. 配位化学国家重点实验室, 人工微结构协同创新中心, 南京大学化学化工学院, 南京 210023

2. 商丘师范学院化学化工学院, 商丘 476000

3. 晋中学院化学化工学院, 晋中 030619

*通讯作者, E-mail: wangxy66@nju.edu.cn

收稿日期: 2020-07-06; 接受日期: 2020-08-04; 网络版发表日期: 2020-10-22

国家重点研究发展计划(编号: 2018YFA0306002)和国家自然科学基金(编号: 21973039)资助项目

摘要分子磁性材料是一类通过化学方法将自由基或顺磁离子及抗磁配体组合而成的具有特定磁学性质的化 合物. 基于三价 $\mathrm{Mo}^{\mathrm{III}}$ 中心的 $\left[\mathrm{Mo}(\mathrm{CN})_{7}\right]^{4-}$ 构筑块在分子磁性材料领域有着独特的研究意义. 一方面, $\mathrm{Mo}^{\mathrm{III}}$ 和其他金 属离子的强磁耦合有利于合成高 $T_{\mathrm{c}}$ 分子基磁体; 另一方面, $\mathrm{Mo}^{\mathrm{III}}$ 的单离子磁各向异性以及和其他金属离子之间的 各向异性磁交换为构筑分子纳米磁体提供了新的思路. 本文总结了基于 $\left[\mathrm{Mo}(\mathrm{CN})_{7}\right]^{4-}$ 构筑块的高维磁体、低维磁 体以及客体调控的磁转换材料, 并对 $\left[\mathrm{Mo}(\mathrm{CN})_{7}\right]^{4-}$ 构筑块在分子磁性材料领域的研究进行了展望.

关键词分子磁性材料, $\left[\mathrm{Mo}(\mathrm{CN})_{7}\right]^{4-}$, 分子基磁体, 各向异性磁交换, 分子纳米磁体

\section{1 引言}

分子磁性材料是一类通过化学方法将自由基或顺 磁离子及抗磁配体组合而成的具有特定磁学性质的化 合物 ${ }^{[1,2]}$. 与基于金属、合金和氧化物等传统磁性材料 相比，分子磁性材料具有体积小、质量轻、易加工和 可塑性强等特点. 由于可以采取“自下而上”的合成策 略以及其有机-无机杂化材料的特点, 分子磁性材料的 结构易于剪裁修饰, 性能易于复合调控. 因此, 它们在 分子自旋电子器件、高密度信息存储、分子开关以及 量子计算等领域具有潜在的应用前景 ${ }^{[3,4]}$.

自旋载体是设计与合成分子磁性材料的物质基 础. 绝大多数分子磁性材料都是以顺磁性过渡金属离 子(包括 $3 \mathrm{~d} 、 4 \mathrm{~d} 、 5 \mathrm{~d} 、 4 \mathrm{f} 、 5 \mathrm{f}$ 离子)为自旋载体. 由于 $3 \mathrm{~d}$
金属离子无论是在实验上还是理论上都比较容易控 制, 一直是分子磁性材料的研究主体. 自从在普鲁士蓝 中发现铁磁相变以来，基于 $3 \mathrm{~d}$ 金属离子的氰基化合物 备受研究人员的关注 ${ }^{[5]}$. 不同金属中心的普鲁士蓝类 似物相继被报道 ${ }^{[6-12]}$, 它们具有非常独特的磁学性能, 如室温磁有序 ${ }^{[7,9]}$ 、光磁行为等 ${ }^{[10 ~ 12]}$. 除此之外, 基于 $4 \mathrm{~d} / 5 \mathrm{~d}$ 金属离子的氰基构筑块也受到了研究人员的高 度关注. 与 $3 \mathrm{~d}$ 金属离子相比, $4 \mathrm{~d} / 5 \mathrm{~d}$ 金属离子具有更加 弥散的 $\mathrm{d}$ 轨道, 这使得它们能够与其他金属离子之间产 生强的磁耦合, 有利于构筑高 $T_{\mathrm{c}}$ 分子基磁体 ${ }^{[13,14]}$. 而且, $4 \mathrm{~d} / 5 \mathrm{~d}$ 金属离子未淬灭的轨道角动量和强的旋轨耦合 使得它们通常具有非常强的磁各向异性，这使得它们 在构筑高性能分子纳米磁体 (单分子磁体及单链磁体) 方面具有很大的潜力 ${ }^{[15]}$. 目前, 广泛研究的 $4 \mathrm{~d} / 5 \mathrm{~d}$ 分子磁

引用格式: Shi L, Wu D, Wei X, Wang X. Research progress in molecular magnetic materials based on the $\left[\mathrm{Mo}(\mathrm{CN})_{7}\right]^{4-}$ unit. Sci Sin Chim, 2020, 50: 1637-1653, doi: 10.1360/SSC-2020-0122 
性材料主要集中于八氧基金属化合物, $\left[\mathrm{M}(\mathrm{CN})_{8}\right]^{3-/ 4-}$ $(\mathrm{M}=\mathrm{Mo}, \mathrm{W}, \mathrm{Nb}, \mathrm{Re})$. 在过去 20 年里, 研究人员制备了 一系列基于八氧根构筑块的磁有序化合物 ${ }^{[16 ~ 20]}$ 、单 分子磁体 ${ }^{[21,22]}$ 、单链磁体 ${ }^{[23 \sim 25]}$ 、自旋转换化合 物 ${ }^{[6-29]}$ 以及多功能磁性化合物 ${ }^{[30 \sim 33]}$. 这些研究结果进 一步体现了 $4 \mathrm{~d} / 5 \mathrm{~d}$ 金属离子作为自旋载体的优越性.

与八氰根金属构筑块相比，基于三价 $\mathrm{Mo}^{\mathrm{III}}$ 中心的 $\left[\mathrm{Mo}(\mathrm{CN})_{7}\right]^{4-}$ 构筑块由于其空气及光照敏感、实验操 作困难, 一直以来的研究相对较少. 然而, 从磁学角度 来讲, 它有着独特的研究意义. 一方面, 理论计算和实 验研究均表明 $\mathrm{Mo}^{\mathrm{III}}$ 和其他金属中心之间具有非常强的 磁耦合，而且易于形成的高维结构使得它有利于制备 高 $T_{\mathrm{c}}$ 分子基磁体. 另一方面, 由于非常强的旋轨耦合, 五角双雉的 $\mathrm{Mo}^{\mathrm{III}}$ 中心具有强的 $g$ 因子各向异性 $\left(g_{z}=3.89, g_{x}=g_{y}=1.77\right)^{[34]} .2003$ 年, 俄罗斯的Mironov研 究组 ${ }^{[35]}$ 通过理论计算指出, $\mathrm{Mo}^{\mathrm{III}}$ 与其他金属离子之间 的磁交换也具有很强的磁各向异性，这种各向异性磁 交换为构筑高能垒和高阻塞温度的分子纳米磁体提供 了可能.

本文总结了文献报道的基于 $\left[\mathrm{Mo}(\mathrm{CN})_{7}\right]^{4-}$ 的高维 磁体、低维磁体以及客体调控的磁转换材料, 并对 $\left[\mathrm{Mo}(\mathrm{CN})_{7}\right]^{4-}$ 构筑块在分子磁性材料领域的研究进行 了展望.

\section{$2\left[\mathrm{Mo}(\mathrm{CN})_{7}\right]^{4-}$ 构筑块}

早在1932年, Young ${ }^{[36]}$ 就第一次合成了 $\mathrm{K}_{4} \mathrm{Mo}-$ $(\mathrm{CN})_{7} \cdot 2 \mathrm{H}_{2} \mathrm{O}$ 构筑块. 1973年, Gray等 ${ }^{[37]}$ 研究了 $\mathrm{K}_{4} \mathrm{Mo}-$ $(\mathrm{CN})_{7} \cdot 2 \mathrm{H}_{2} \mathrm{O}$ 固体和溶液中的红外光谱和拉曼光谱. 1980年，Malik等 ${ }^{[38]}$ 利用单晶X衍射技术表征了 $\mathrm{NaK}_{3}$ $\mathrm{Mo}(\mathrm{CN})_{7} \cdot 2 \mathrm{H}_{2} \mathrm{O}$ 和 $\mathrm{K}_{4} \mathrm{Mo}(\mathrm{CN})_{7} \cdot 2 \mathrm{H}_{2} \mathrm{O}$ 的晶体结构. 研究 发现, 7 个㲵根基团配位的 $\mathrm{Mo}^{\mathrm{III}}$ 中心主要存在两种配 位构型：五角双雉(PBP)和单帽三棱柱(CTP). 其中, $\mathrm{NaK}_{3} \mathrm{Mo}(\mathrm{CN})_{7} \cdot 2 \mathrm{H}_{2} \mathrm{O}$ 和 $\mathrm{K}_{4} \mathrm{Mo}(\mathrm{CN})_{7} \cdot 2 \mathrm{H}_{2} \mathrm{O}$ 水溶液中的 $\mathrm{Mo}^{\mathrm{III}}$ 具有五角双雉的几何结构, 而固态的 $\mathrm{K}_{4} \mathrm{Mo}$ $(\mathrm{CN})_{7} \cdot 2 \mathrm{H}_{2} \mathrm{O}$ 中的 $\mathrm{Mo}^{\mathrm{III}}$ 则具有单帽三棱柱的几何结构. $\mathrm{Mo}^{\mathrm{III}}$ 中心不同的配位构型导致其配体场能级图和基态 电子构型也有很大的差异(图1). 对于五角双雉的 $\mathrm{Mo}^{\mathrm{III}}$ 中心, $D_{5 h}$ 对称性产生 ${ }^{2} \mathrm{E}_{1}-\left(\mathrm{e}_{1}{ }^{\prime}\right)^{3}$ 的基态电子构型; 而单 帽三棱柱具有 $C_{2 v}$ 的对称性, 其配体场能级分裂产 生 ${ }^{2} \mathrm{~A}_{1}-\left(\mathrm{a}_{2}\right)^{2}\left(\mathrm{a}_{1}\right)^{1}$ 基态电子构型. 不同的基态电子构型可

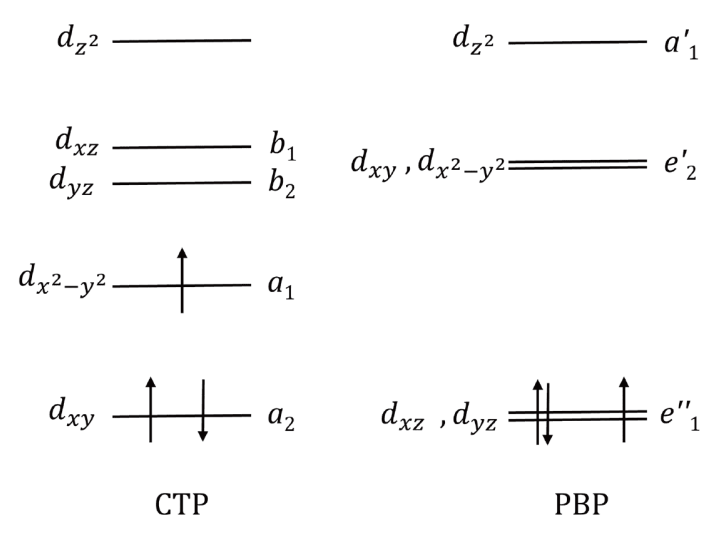

图 $1 \mathrm{Mo}^{\mathrm{III}}$ 处于单帽三棱柱 $(\mathrm{CTP})$ 和五角双锥 $(\mathrm{PBP})$ 构型中 配体场能级图和基态电子构型

Figure 1 Ligand field energy level diagrams and ground-state electronic configurations for monocapped trigonal-prismatic (CTP) and pentagonal-bipyramidal (PBP) geometry of $\mathrm{Mo}^{\mathrm{III}}$.

进一步通过红外光谱和拉曼光谱确定. 此外, 电子顺磁 共振(EPR)测量显示固态的 $\mathrm{K}_{4} \mathrm{Mo}(\mathrm{CN})_{7} \cdot 2 \mathrm{H}_{2} \mathrm{O}$ 的 $g$ 因子 分别为: $g_{z}=2.103, g_{x}=g_{y}=1.97$, 这个结果也与具有 $C_{2 v}$ 对 称性产生的 ${ }^{2} \mathrm{~A}_{1}\left(\mathrm{a}_{2}\right)^{2}\left(\mathrm{a}_{1}\right)^{1}$ 基态电子构型计算的 $g$ 因子保 持一致 ( $\left.g_{z}=2.103, g_{x}=1.983, g_{y}=1.984\right)$, 这和 $\mathrm{K}_{4} \mathrm{Mo}$ $(\mathrm{CN})_{7} \cdot 2 \mathrm{H}_{2} \mathrm{O}$ 固体中的 $\mathrm{Mo}^{\mathrm{III}}$ 的单帽三棱柱的几何结构相 吻合 ${ }^{[37]}$.

由于五角双雉的配位构型和单帽三棱柱配位构型 相互转换过程中几乎没有能量势垒, 通过改变 $\left[\mathrm{Mo}(\mathrm{CN})_{7}\right]^{4-}$ 阴离子的外部环境, 如不同的阳离子和客 体分子就能改变 $\mathrm{Mo}^{\mathrm{III}}$ 中心的配位构型. 因此, 柔性的 $\left[\mathrm{Mo}(\mathrm{CN})_{7}\right]^{4-}$ 单元非常有利于构筑不同拓扑结构的磁 性配合物(表1).

\section{3 基于 $\left[\mathrm{Mo}(\mathrm{CN})_{7}\right]^{4-}$ 的高维磁体}

尽管 $\mathrm{K}_{4} \mathrm{Mo}(\mathrm{CN})_{7} \cdot 2 \mathrm{H}_{2} \mathrm{O}$ 在1932年就已经被报道, 但 由于其空气及光照敏感性, 利用其来构筑分子磁性材 料的研究却一直没有开展. 1998年, Kahn等 ${ }^{[39]}$ 报道了 第一例基于 $\left[\mathrm{Mo}(\mathrm{CN})_{7}\right]^{4-}$ 的分子基磁体, 并引起了研究 人员对基于 $\left[\mathrm{Mo}(\mathrm{CN})_{7}\right]^{4-}$ 的分子磁性材料的关注. 实验 上, 由于 $\left[\mathrm{Mo}(\mathrm{CN})_{7}\right]^{4-}$ 高的负电荷以及数量众多的氰基 基团, 其很容易和 $3 \mathrm{~d}$ 金属离子发生反应而产生沉淀, 不 利于获得配合物的单晶并进行详细的结构-性能相关 研究. 为了解决这一难题, 研究人员采用添加辅助配体 或引入额外阳离子的合成策略. 一方面, 通过使用辅助 
表 1 基于 $\left[\mathrm{Mo}(\mathrm{CN})_{7}\right]^{4-}$ 的分子磁性材料

Table 1 Molecular magnetic materials based on $\left[\mathrm{Mo}(\mathrm{CN})_{7}\right]^{4-}$

\begin{tabular}{|c|c|c|c|c|c|}
\hline 化合物 & 结构 & $\mathrm{Mo}^{\mathrm{III}}$ 的构型 & $T_{\mathrm{c}}$ 或 $T_{B}(\mathrm{~K})$ & 矫顽场 $H_{\mathrm{c}}(\mathrm{Oe})$ & 参考文献 \\
\hline$\left\{\mathrm{Mn}_{2}\left(\mathrm{H}_{2} \mathrm{O}\right)_{5}\left[\mathrm{Mo}(\mathrm{CN})_{7}\right] \cdot 4 \mathrm{H}_{2} \mathrm{O}\right\}_{n}$ & $3 \mathrm{D}$ & PBP & 51 & $0^{(5 \mathrm{~K}), \mathrm{a})}$ & {$[39]$} \\
\hline$\left\{\mathrm{Mn}_{2}\left(\mathrm{H}_{2} \mathrm{O}\right)_{5}\left[\mathrm{Mo}(\mathrm{CN})_{7}\right] \cdot 4.75 \mathrm{H}_{2} \mathrm{O}\right\}_{n}$ & $3 \mathrm{D}$ & СТP & 51 & $0^{(5 \mathrm{~K})}$ & [40] \\
\hline$\left\{\mathrm{K}_{2}\left[\mathrm{Mn}\left(\mathrm{H}_{2} \mathrm{O}\right)_{2}\right]_{3}\left[\mathrm{Mo}(\mathrm{CN})_{7}\right]_{2} \cdot 6 \mathrm{H}_{2} \mathrm{O}\right\}_{n}$ & $2 \mathrm{D}$ & СТP & 39 & $0^{(5 \mathrm{~K})}$ & {$[44]$} \\
\hline$\left\{\left[\mathrm{N}\left(\mathrm{CH}_{3}\right)_{4}\right]_{2}\left[\mathrm{Mn}\left(\mathrm{H}_{2} \mathrm{O}\right)\right]_{3}\left[\mathrm{Mo}(\mathrm{CN})_{7}\right]_{2} \cdot 2 \mathrm{H}_{2} \mathrm{O}\right\}_{n}$ & $3 \mathrm{D}$ & PBP & 86 & $0^{(5 \mathrm{~K})}$ & {$[46]$} \\
\hline$\left\{\left(\mathrm{NH}_{4}\right)_{2}\left[\mathrm{Mn}_{3}\left(\mathrm{H}_{2} \mathrm{O}\right)_{4}\right]_{3}\left[\mathrm{Mo}(\mathrm{CN})_{7}\right]_{2} \bullet n \mathrm{H}_{2} \mathrm{O}\right\}_{n}$ & $3 \mathrm{D}$ & СТP & 53 & $0^{(5 \mathrm{~K})}$ & {$[47]$} \\
\hline$\left\{[\mathrm{Mn}(\mathrm{dpop})]_{6}\left[\mathrm{Mo}(\mathrm{CN})_{7}\right]\left[\mathrm{Mo}(\mathrm{CN})_{8}\right]_{2} \cdot 19.5 \mathrm{H}_{2} \mathrm{O}\right\}_{n}$ & $2 \mathrm{D}$ & PBP & 3 & $0^{(1.8 \mathrm{~K})}$ & [49] \\
\hline$\left\{[\mathrm{Mn}(\operatorname{tacn})]_{2}\left[\mathrm{Mo}(\mathrm{CN})_{7}\right] \cdot 5 \mathrm{H}_{2} \mathrm{O}\right\}_{n}$ & $3 \mathrm{D}$ & & 90 & $0^{(5 \mathrm{~K})}$ & {$[50]$} \\
\hline$\left\{\left[\mathrm{Mn}_{2}(\text { tea }) \mathrm{Mo}(\mathrm{CN})_{7}\right] \cdot \mathrm{H}_{2} \mathrm{O}\right\}_{n}$ & $3 \mathrm{D}$ & CTP & 75 & $70^{(5 \mathrm{~K})}$ & [51] \\
\hline$\left\{\left[\mathrm{Mn}_{2}(\text { tea }) \mathrm{Mo}(\mathrm{CN})_{7}\right]\right\}_{n}$ & $3 \mathrm{D}$ & СТР & 106 & $200^{(5 \mathrm{~K})}$ & [51] \\
\hline$\left\{\mathrm{Mn}^{\mathrm{II}}\left[\mathrm{Mn}^{\mathrm{II}}(\mathrm{Hdman})\right]_{2}\left[\mathrm{Mo}^{\mathrm{III}}(\mathrm{CN})_{7}\right]_{2} \cdot 2 \mathrm{H}_{2} \mathrm{O}\right\}_{n}$ & $3 \mathrm{D}$ & СТP & 106 & $150^{(5 \mathrm{~K})}$ & {$[52]$} \\
\hline$\left\{\mathrm{Mn}_{2}\left[\mathrm{Mo}^{\mathrm{III}}(\mathrm{CN})_{7}\right] \cdot(\text { pyrimidine })_{2} \cdot 2 \mathrm{H}_{2} \mathrm{O}\right\}_{n}$ & $3 \mathrm{D}$ & $\mathrm{MCO}$ & 47 & $60^{(2 \mathrm{~K})}$ & [53] \\
\hline$\left\{\mathrm{V}_{2}^{\mathrm{II}}\left[\mathrm{Mo}^{\mathrm{III}}(\mathrm{CN})_{7}\right] \cdot(\text { pyrimidine })_{2} \cdot 2 \mathrm{H}_{2} \mathrm{O}\right\}_{n}$ & $3 \mathrm{D}$ & $\mathrm{MCO}$ & 110 & $300^{(2 \mathrm{~K})}$ & {$[53]$} \\
\hline$\left\{[\mathrm{Mn}(\mathrm{dpop})]_{3}\left[\mathrm{Mn}(\mathrm{dpop})\left(\mathrm{H}_{2} \mathrm{O}\right)\right]\left[\mathrm{Mo}(\mathrm{CN})_{7}\right]_{2} \cdot 13.5 \mathrm{H}_{2} \mathrm{O}\right\}_{n}$ & $3 \mathrm{D}$ & PBP & 2.6 & $90^{(1.8 \mathrm{~K})}$ & {$[54]$} \\
\hline$\left\{\left[\mathrm{Mn}(\mathrm{dpop})_{2}\right]\left[\mathrm{Mo}(\mathrm{CN})_{7}\right] \cdot 2 \mathrm{H}_{2} \mathrm{O}\right\}_{n}$ & $3 \mathrm{D}$ & PBP & 24 & $305^{(1.8 \mathrm{~K})}$ & {$[54]$} \\
\hline$\left\{[\mathrm{Mn}(\mathrm{dpop})]_{4}\left[(\mathrm{dpop}) \mathrm{Mn}\left(\mathrm{H}_{2} \mathrm{O}\right)\right]_{2}\left[\mathrm{Mo}(\mathrm{CN})_{7}\right]_{3} \cdot 27 \mathrm{H}_{2} \mathrm{O}\right\}_{n}$ & $2 \mathrm{D}$ & PBP & 2.2 & $30^{(1.8 \mathrm{~K})}$ & {$[55]$} \\
\hline$\left\{\mathrm{Mn}_{2}(1-\text { pypz })\left(\mathrm{H}_{2} \mathrm{O}\right)\left(\mathrm{CH}_{3} \mathrm{CN}\right)\left[\mathrm{Mo}(\mathrm{CN})_{7}\right]\right\}_{n}$ & $3 \mathrm{D}$ & СТP & 66 & $820^{(2 \mathrm{~K})}$ & {$[56]$} \\
\hline$\left\{\mathrm{Mn}_{2}(3-\mathrm{pypz})\left(\mathrm{H}_{2} \mathrm{O}\right)\left(\mathrm{CH}_{3} \mathrm{CN}\right)\left[\mathrm{Mo}(\mathrm{CN})_{7}\right]\right\}_{n}$ & $3 \mathrm{D}$ & CTP & 64 & $72^{(2 \mathrm{~K})}$ & {$[56]$} \\
\hline$\left\{\mathrm{Mn}_{2}(\text { pyim })\left(\mathrm{H}_{2} \mathrm{O}\right)\left(\mathrm{CH}_{3} \mathrm{CN}\right)\left[\mathrm{Mo}(\mathrm{CN})_{7}\right]\right\}_{n}$ & $3 \mathrm{D}$ & CTP & 62 & $146^{(2 \mathrm{~K})}$ & {$[56]$} \\
\hline$\left\{\left(\mathrm{NH}_{4}\right)_{3}\left[\left(\mathrm{H}_{2} \mathrm{O}\right) \mathrm{Mn}_{3}(\mathrm{HCOO})\right]\left[\mathrm{Mo}(\mathrm{CN})_{7}\right]_{2} \cdot 4 \mathrm{H}_{2} \mathrm{O}\right\}_{n}$ & $3 \mathrm{D}$ & PBP & 70 & $1500^{(2 \mathrm{~K})}$ & {$[57]$} \\
\hline$\left\{\mathrm{Mn}_{2}(\mathrm{DMF})\left(\mathrm{H}_{2} \mathrm{O}\right)_{2}\left[\mathrm{Mo}(\mathrm{CN})_{7}\right] \cdot \mathrm{H}_{2} \mathrm{O} \cdot \mathrm{CH}_{3} \mathrm{OH}\right\}_{n}$ & $3 \mathrm{D}$ & СТP & 80 & $250^{(2 \mathrm{~K})}$ & {$[58]$} \\
\hline$\left\{\mathrm{Mn}_{2}(\mathrm{DEF})\left(\mathrm{H}_{2} \mathrm{O}\right)\left[\mathrm{Mo}(\mathrm{CN})_{7}\right]\right\}_{n}$ & $3 \mathrm{D}$ & СТP & 80 & $200^{(2 \mathrm{~K})}$ & {$[58]$} \\
\hline$\left\{\left[\mathrm{Mn}(\mathrm{imH})_{2}\right]_{2}\left[\mathrm{Mn}\left(\mathrm{H}_{2} \mathrm{O}\right)(\mathrm{imH})_{3}\right]\left[\mathrm{Mn}(\mathrm{imH})_{4}\right]\left[\mathrm{Mo}(\mathrm{CN})_{7}\right]_{2} \cdot 6 \mathrm{H}_{2} \mathrm{O}\right\}_{n}$ & $3 \mathrm{D}$ & PBP & 29 & $5000^{(1.8 \mathrm{~K})}$ & [59] \\
\hline$\left\{\left[\mathrm{Mn}\left(\mathrm{H}_{2} \mathrm{O}\right)_{2}(\mathrm{imH})\right]_{3}\left[\mathrm{Mn}\left(\mathrm{H}_{2} \mathrm{O}\right)(\mathrm{imH})_{2}\right]\left[\mathrm{Mo}(\mathrm{CN})_{7}\right]_{2} \cdot 5 \mathrm{H}_{2} \mathrm{O}\right\}_{n}$ & $3 \mathrm{D}$ & PBP & 45 & $4500^{(1.8 \mathrm{~K})}$ & [59] \\
\hline$\left\{\left[\mathrm{Mn}(\mathrm{Htrz})\left(\mathrm{H}_{2} \mathrm{O}\right)_{2}\right]\left[\mathrm{Mn}(\mathrm{Htrz})_{0.7}\left(\mathrm{H}_{2} \mathrm{O}\right)_{2.3}\right]\left[\mathrm{Mo}(\mathrm{CN})_{7}\right] \cdot 5.6 \mathrm{H}_{2} \mathrm{O}\right\}_{n}$ & $3 \mathrm{D}$ & PBP & 45 & $710^{(1.8 \mathrm{~K})}$ & [59] \\
\hline$\left\{\left[\mathrm{Mn}\left(\mathrm{H}_{2} \mathrm{O}\right)_{2}\right]_{3}\left[\mathrm{Mn}\left(\mathrm{H}_{2} \mathrm{O}\right)_{4}\right]\left[\mathrm{Mo}(\mathrm{CN})_{7}\right]_{2} \cdot 6 \mathrm{H}_{2} \mathrm{O} \cdot 2 \text { urea }\right\}_{n}$ & $3 \mathrm{D}$ & PBP & 59 & $110^{(1.8 \mathrm{~K})}$ & [59] \\
\hline$\left\{\mathrm{Fe}_{2}\left(\mathrm{H}_{2} \mathrm{O}\right)_{5}\left[\mathrm{Mo}(\mathrm{CN})_{7}\right] \cdot 5 \mathrm{H}_{2} \mathrm{O}\right\}_{n}$ & $3 \mathrm{D}$ & СТP & 65 & $6000^{(2 \mathrm{~K})}$ & {$[61]$} \\
\hline$\left\{\left[\mathrm{NH}_{2}\left(\mathrm{CH}_{3}\right)_{2}\right]_{2} \mathrm{Fe}_{5}\left(\mathrm{H}_{2} \mathrm{O}\right)_{10}\left[\mathrm{Mo}(\mathrm{CN})_{7}\right]_{3} \cdot 8 \mathrm{H}_{2} \mathrm{O}\right\}_{n}$ & $3 \mathrm{D}$ & СТP & 65 & $1900^{(2 \mathrm{~K})}$ & {$[61]$} \\
\hline $\mathrm{K}_{0.6} \mathrm{Ni}_{1.7}\left[\mathrm{Mo}(\mathrm{CN})_{7}\right] \cdot 5.5 \mathrm{H}_{2} \mathrm{O} \cdot 0.5 \mathrm{CH}_{3} \mathrm{OH}$ & $3 \mathrm{D}$ & СТP & 28 & $500^{(2 \mathrm{~K})}$ & {$[62]$} \\
\hline$\left[\mathrm{Mn}(\text { dpop })\left(\mathrm{H}_{2} \mathrm{O}\right)_{2}\right]_{2}\left[\left\{\mathrm{Mo}(\mathrm{CN})_{7}\right\}_{8}\{\mathrm{Mn}(\text { dpop })\}_{10}\left\{\mathrm{Mn}(\text { dpop })\left(\mathrm{H}_{2} \mathrm{O}\right)\right\}_{4}\right] \cdot x \mathrm{H}_{2} \mathrm{O}$ & $0 \mathrm{D}$ & PBP & 3.6 & $180^{(1.8 \mathrm{~K})}$ & {$[63]$} \\
\hline$\left[\mathrm{Mo}^{\text {III }}(\mathrm{CN})_{8}\right]_{6}[\mathrm{Ni}(\mathrm{dtpd})]_{12}\left(\mathrm{H}_{2} \mathrm{O}\right)_{6}$ & $0 \mathrm{D}$ & СТP & 0 & $0^{(1.8 \mathrm{~K})}$ & {$[64]$} \\
\hline$\left[\mathrm{Mn}\left(\mathrm{L}_{\mathrm{N} 5 \mathrm{Me}}\right) \mathrm{H}_{2} \mathrm{O}\right]_{2}\left[\mathrm{Mo}(\mathrm{CN})_{7}\right] \cdot 6 \mathrm{H}_{2} \mathrm{O}$ & $0 \mathrm{D}$ & PBP & 3.2 & $20000^{(1.8 \mathrm{~K})}$ & {$[65]$} \\
\hline$\left[\mathrm{Mn}\left(\mathrm{L}_{\mathrm{N} 3 \mathrm{O} 2}\right)\left(\mathrm{H}_{2} \mathrm{O}\right)\right]_{2}\left[\mathrm{Mo}(\mathrm{CN})_{7}\right] \cdot 7 \mathrm{H}_{2} \mathrm{O}$ & $0 \mathrm{D}$ & PBP & 0 & $0^{(1.8 \mathrm{~K})}$ & {$[65]$} \\
\hline$\left[\mathrm{Mn}\left(\mathrm{L}_{\mathrm{DAPSC}}\right)\left(\mathrm{H}_{2} \mathrm{O}\right)\right]_{2}\left[\mathrm{Mo}(\mathrm{CN})_{7}\right] \cdot 6 \mathrm{H}_{2} \mathrm{O} \cdot \mathrm{CH}_{3} \mathrm{CN}$ & $0 \mathrm{D}$ & PBP & 0 & $0^{(1.8 \mathrm{~K})}$ & {$[65]$} \\
\hline$\left\{\left[\mathrm{Mn}\left(\mathrm{L}_{\mathrm{N} 5 \mathrm{C} 10}\right)\right]_{2}\left[\mathrm{Mo}(\mathrm{CN})_{7}\right] \cdot 2 \mathrm{H}_{2} \mathrm{O}\right\}_{n}$ & $1 \mathrm{D}$ & PBP & 5.6 & $1150^{(21.8 \mathrm{~K})}$ & {$[67]$} \\
\hline$\left\{[\mathrm{Mn}(\text { dpop })]\left[\mathrm{Mn}(\text { dpop })\left(\mathrm{H}_{2} \mathrm{O}\right)\right]\left[\mathrm{Mo}(\mathrm{CN})_{7}\right] \cdot 7.5 \mathrm{H}_{2} \mathrm{O}\right\}_{n}$ & $1 \mathrm{D}$ & PBP & 3.2 & $0^{(1.8 \mathrm{~K})}$ & {$[67]$} \\
\hline$\left\{[\mathrm{Mn}(\text { dpop })]\left[\mathrm{Mn}(\text { dpop })\left(\mathrm{H}_{2} \mathrm{O}\right)\right]\left[\mathrm{Mo}(\mathrm{CN})_{7}\right] \cdot 9 \mathrm{H}_{2} \mathrm{O}\right\}_{n}$ & $1 \mathrm{D}$ & PBP & 0 & $0^{(1.8 \mathrm{~K})}$ & {$[67]$} \\
\hline $\begin{array}{c}\left\{[\mathrm{Mn}(\mathrm{dpop})]\left[\mathrm{Mn}(\mathrm{dpop})\left(\mathrm{H}_{2} \mathrm{O}\right)\right]\left[\mathrm{Mo}(\mathrm{CN})_{7}\right][\mathrm{Mn}(\mathrm{dpop})]\left[\mathrm{Mn}(\mathrm{dpop})\left(\mathrm{H}_{2} \mathrm{O}\right)\right][\mathrm{Mo}\right. \\
\left.\left.(\mathrm{CN})_{7}\right] \cdot 23 \mathrm{H}_{2} \mathrm{O}\right\}_{n}\end{array}$ & $1 \mathrm{D}$ & PBP & 2.0 & $0^{(1.8 \mathrm{~K})}$ & {$[68]$} \\
\hline$\left\{\left[\mathrm{Mn}(\text { bida })\left(\mathrm{H}_{2} \mathrm{O}\right)\right]_{2}\left[\mathrm{Mo}(\mathrm{CN})_{7}\right] \cdot 6 \mathrm{H}_{2} \mathrm{O}\right\}_{n}$ & $1 \mathrm{D}$ & PBP & 5.8 & $15000^{(2.0 \mathrm{~K})}$ & [69] \\
\hline
\end{tabular}

a) 表示在温度为 $5 \mathrm{~K}$ 时未观察到磁滞回线 
配体与 $3 \mathrm{~d}$ 金属离子配位，可以减少和 $3 \mathrm{~d}$ 金属离子配位 的氧基数目. 另一方面, 通过引入额外的抗衡阳离子, 可以降低 $3 \mathrm{~d}$ 金属离子和 $\left[\mathrm{Mo}(\mathrm{CN})_{7}\right]^{4-}$ 单元的比例. 这些 合成策略不仅能够降低产生沉淀的速度, 还可能改变 所形成的配合物的拓扑结构, 从而达到改变其有序温 度及磁学性质的目的.

在过去 20 年里, 研究人员报道了一系列包含不同 $3 \mathrm{~d}$ 金属中心(如 $\mathrm{Mn}^{\mathrm{II}} 、 \mathrm{Fe}^{\mathrm{II}} 、 \mathrm{Ni}^{\mathrm{II}}$ 和 $\mathrm{V}^{\mathrm{II}}$ ) 的 $\left[\mathrm{Mo}(\mathrm{CN})_{7}\right]^{4-}$ 分 子磁性材料. 由于存在 7 个㲵根基团, 这些化合物通常 结晶在较低对称性的空间群中. 而且, 由于有些化合物 可以获得较大的单晶, 研究者通过单晶磁性测量详细 研究了它们的单晶磁性. 这些研究结果为获得基于 $\left[\mathrm{Mo}(\mathrm{CN})_{7}\right]^{4-}$ 的高 $T_{\mathrm{c}}$ 分子基磁体提供了思路.

\section{1 最早的 $\mathrm{Mn}^{\mathrm{II}}-\mathrm{Mo}^{\mathrm{III}}$ 三维磁体: $\boldsymbol{\alpha}$ 相和 $\boldsymbol{\beta}$ 相}

1998年，Kahn等使用 $\left[\mathrm{Mo}(\mathrm{CN})_{7}\right]^{4-}$ 和 $\mathrm{Mn}$ 吕离子在水 中通过缓慢扩散自组装获得了两个热稳定的晶体 $\left\{\mathrm{Mn}_{2}\left(\mathrm{H}_{2} \mathrm{O}\right)_{5}\left[\mathrm{Mo}^{\mathrm{III}}(\mathrm{CN})_{7}\right] \bullet 4 \mathrm{H}_{2} \mathrm{O}\right\}_{n} \quad(\alpha \text { 相 })^{[39]}$ 和 $\left\{\mathrm{Mn}_{2}-\right.$ $\left.\left(\mathrm{H}_{2} \mathrm{O}\right)_{5}\left[\mathrm{Mo}(\mathrm{CN})_{7}\right] \cdot 4.75 \mathrm{H}_{2} \mathrm{O}\right\}_{n}(\beta \text { 相 })^{[40]}$. 两个化合物的

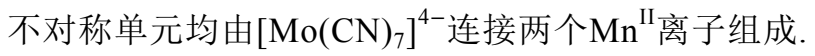
尽管两个化合物的分子式极为相似, 它们的结构却完 全不同. 在 $\alpha$ 相结构中, $\mathrm{Mo}^{\mathrm{III}}$ 中心处于五角双雉的配位

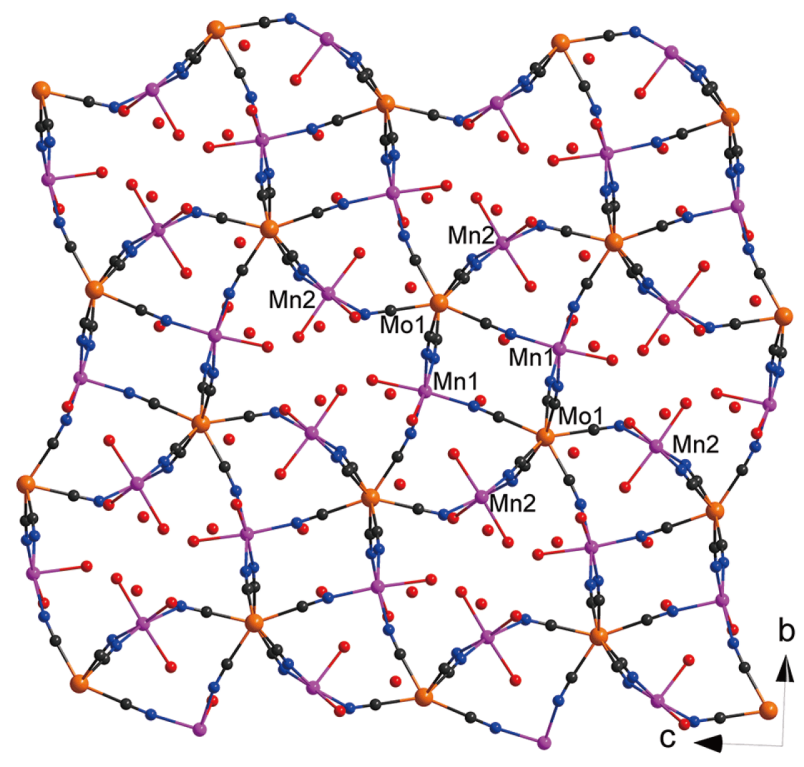

图 $2\left\{\mathrm{Mn}_{2}\left(\mathrm{H}_{2} \mathrm{O}\right)_{5}\left[\mathrm{Mo}^{\mathrm{III}}(\mathrm{CN})_{7}\right] \cdot 4 \mathrm{H}_{2} \mathrm{O}\right\}_{n}(\alpha$ 相)沿着 $a$ 方向的晶 体结构图. 为了简洁, 省略了氢原子 ${ }^{[39]}$ (网络版彩图)

Figure 2 Crystal structure of the compound $\left\{\mathrm{Mn}_{2}\left(\mathrm{H}_{2} \mathrm{O}\right)_{5}\left[\mathrm{Mo}^{\mathrm{III}}(\mathrm{CN})_{7}\right]\right.$ $\left.-4 \mathrm{H}_{2} \mathrm{O}\right\}_{n}$ ( $\alpha$ phase) viewed along the $a$ direction. Hydrogen atoms have been omitted for clarity [39] (color online).
环境( $\mathrm{PBP})$, 并且通过 7 个氭根基团连接 $\mathrm{Mn}^{\mathrm{II}}$ 离子. 如图 2所示, 每个 $\left[\mathrm{Mo}(\mathrm{CN})_{7}\right]^{4-}$ 单元通过两个氧根基团连接两 个Mn1, 形成Mo1-Mn1-Mo1-Mn1四方单元. 这些四方单 元进一步通过氰根基团连接并沿着 $a$ 轴方向形成弯曲的 梯型链. 每个梯型链通过四个 Mn2位点连接成三维框架.

对于 $\beta$ 相, $\mathrm{Mo}^{\mathrm{III}}$ 中心的配位构型与 $\alpha$ 相截然不同, 处 于单帽三棱柱配位环境中. $\beta$ 相的三维结构可以看作由 平行的格子状二维层通过Mn2离子连成三维配位框架 (图3). 这种配位组装导致三维结构沿着 $b$ 轴方向产生 了一维的孔道, 其中填充了结晶水分子.

无论是 $\alpha$ 相还是 $\beta$ 相，配位水分子以及结晶水分子 通过氢键相互作用稳定 $\mathrm{Mo}^{\mathrm{III}}-\mathrm{Mn}^{\mathrm{II}}$ 三维框架. 因此, 两 个化合物均具有较高的热稳定性. 由于这两个化合物 能够获得较大的单晶, Kahn等 ${ }^{[39]}$ 对其进行详细的单晶 磁测量. 测量结果表明 $\alpha$ 相和 $\beta$ 相的磁有序温度均达到 $51 \mathrm{~K}$ ，并且在低温下具有自旋重排行为，其磁有序的 磁矩方向发生重新排列, 在更低温度的交流磁化率上 出现新峰 ${ }^{[41]}$. 更为有趣的是, 两个化合物均呈现出场 致自旋取向现象，表明 $\alpha$ 相和 $\beta$ 相中强的磁各向异性 ${ }^{[42]}$. 这种磁各向异性主要源于三价Mo中心强的自旋轨道

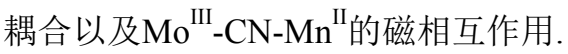

与高对称性的普鲁士蓝分子基磁体相比, 各向异 性的Mo-Mn三维框架使得这两个化合物均具有罕见

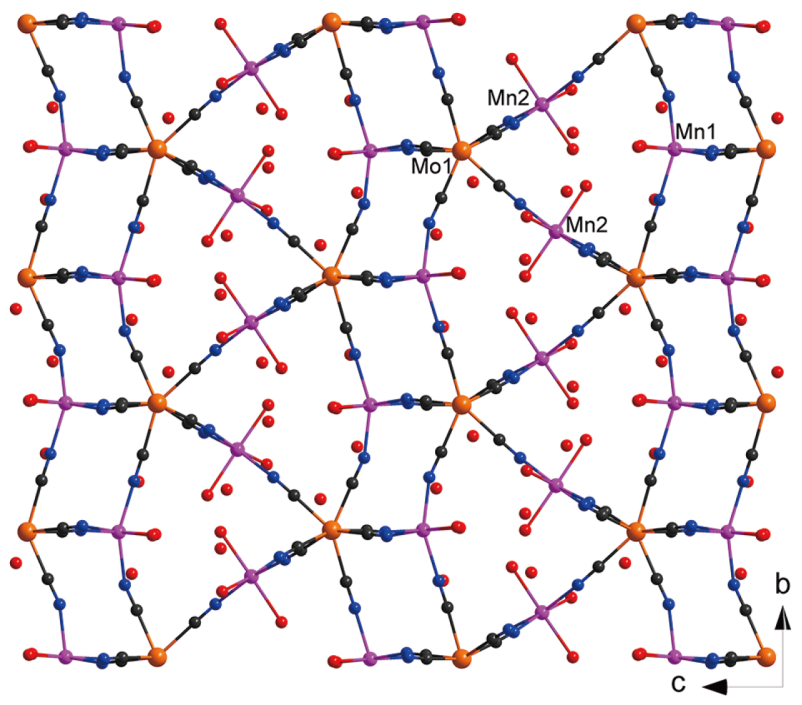

图 $3\left\{\mathrm{Mn}_{2}\left(\mathrm{H}_{2} \mathrm{O}\right)_{5}\left[\mathrm{Mo}{ }^{\mathrm{III}}(\mathrm{CN})_{7}\right] \bullet 4.75 \mathrm{H}_{2} \mathrm{O}\right\}_{n}(\beta$ 相 $)$ 沿着 $a$ 方向的 晶体结构图. 为了简洁, 省略了氢原子 ${ }^{[39]}$ (网络版彩图) Figure 3 Crystal structure of the compound $\left\{\mathrm{Mn}_{2}\left(\mathrm{H}_{2} \mathrm{O}\right)_{5}\left[\mathrm{Mo}{ }^{\text {III }}(\mathrm{CN})_{7}\right]\right.$ $\left.\cdot 4.75 \mathrm{H}_{2} \mathrm{O}\right\}_{n}(\beta$ phase $)$ viewed along the a direction. Hydrogen atoms have been omitted for clarity [39] (color online). 
的自旋重排现象. 而且这两个化合物在当时具有相对 较高的磁有序温度．这表明有望通过不同的合成策略 构筑基于 $\left[\mathrm{Mo}(\mathrm{CN})_{7}\right]^{4-}$ 的低对称性高维化合物, 来获得 高 $T_{\mathrm{c}}$ 分子磁性材料 ${ }^{[43]}$.

\section{2 阳离子取代策略构筑 $\mathrm{Mn}^{\mathrm{II}}-\mathrm{Mo}^{\mathrm{III}}$ 高 $\boldsymbol{T}_{\mathrm{c}}$ 磁体}

通过将 $\left[\mathrm{Mo}(\mathrm{CN})_{7}\right]^{4-}$ 与 $\mathrm{Mn}^{\mathrm{II}}$ 离子在饱和的 $\mathrm{KNO}_{3}$ 溶 液中自组装，Larionova等 ${ }^{[44]}$ 获得了一例二维层状化合 物 $\left\{\mathrm{K}_{2}\left[\mathrm{Mn}\left(\mathrm{H}_{2} \mathrm{O}\right)_{2}\right]_{3}\left[\mathrm{Mo}(\mathrm{CN})_{7}\right]_{2} \cdot 6 \mathrm{H}_{2} \mathrm{O}\right\}_{n}$. 该化合物中, $\mathrm{Mo}{ }^{\mathrm{III}}$ 中心处于单帽三棱柱配位构型，使用 6 个氭根基 团与 $\mathrm{Mn}^{\mathrm{II}}$ 离子配位， $\mathrm{Mn}^{\mathrm{II}}$ 离子均处于八面体配位构型 中. $\mathrm{Mn}-\mathrm{Mo}$ 通过氰根桥连形成平行于 $b c$ 平面的双层结 构, $\mathrm{K}^{+}$离子和结晶水分子分布于相邻的两个层之间(图 4). 与 $\alpha$ 相和 $\beta$ 相化合物相似，由于存在磁各向异性，该 化合物也能够观察到场致的自旋取向现象 ${ }^{[45]}$, 其磁有 序温度为 $39 \mathrm{~K}$. 较低的磁有序温度可能与化合物相对 较低的结构维数有关.

除了 $\mathrm{K}^{+}$外，Larionova等 ${ }^{[46]}$ 使用 $\left[\mathrm{N}\left(\mathrm{CH}_{3}\right)_{4}\right] \mathrm{Cl}$ 饱和溶 液代替 $\mathrm{KNO}_{3}$ 饱和溶液，合成了一例含 $\left[\mathrm{N}\left(\mathrm{CH}_{3}\right)_{4}\right]^{+}$离子 的 $\mathrm{Mo}^{\mathrm{III}}-\mathrm{Mn}^{\mathrm{II}}$ 化合物一 $-\left\{\left[\mathrm{N}\left(\mathrm{CH}_{3}\right)_{4}\right]_{2}\left[\mathrm{Mn}\left(\mathrm{H}_{2} \mathrm{O}\right)\right]_{3}[\mathrm{Mo}-\right.$ $\left.\left.(\mathrm{CN})_{7}\right]_{2} \cdot 2 \mathrm{H}_{2} \mathrm{O}\right\}_{n}$. 尽管 $\left[\mathrm{N}\left(\mathrm{CH}_{3}\right)_{4}\right]^{+}$离子具有更大的尺寸, 但是该阳离子嵌入到 $\mathrm{Mo}^{\mathrm{III}}-\mathrm{Mn}^{\mathrm{II}}$ 配位框架的孔道中，形

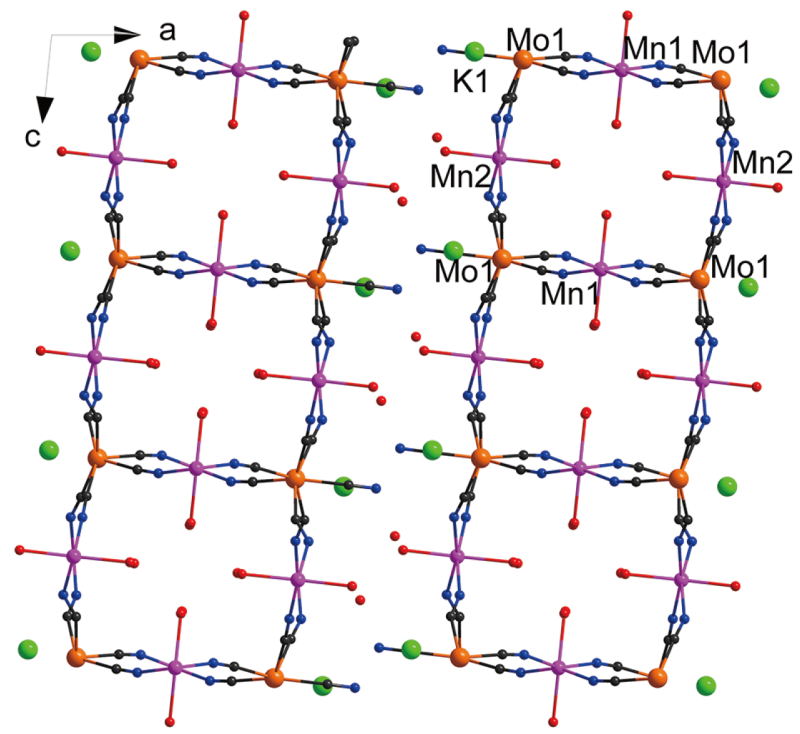

图 $4\left\{\mathrm{~K}_{2}\left[\mathrm{Mn}\left(\mathrm{H}_{2} \mathrm{O}\right)_{2}\right]_{3}\left[\mathrm{Mo}(\mathrm{CN})_{7}\right]_{2} \cdot 6 \mathrm{H}_{2} \mathrm{O}\right\}_{n}$ 在 $a c$ 面的晶体结 构图. 为了简洁, 省略了氢原子 ${ }^{[44]}$ (网络版彩图)

Figure 4 Crystal structure of the compound $\left\{\mathrm{K}_{2}\left[\mathrm{Mn}\left(\mathrm{H}_{2} \mathrm{O}\right)_{2}\right]_{3}[\mathrm{Mo}-\right.$ $\left.\left.(\mathrm{CN})_{7}\right]_{2} \cdot 6 \mathrm{H}_{2} \mathrm{O}\right\}_{n}$ in the ac plane. Hydrogen atoms have been omitted for clarity [44] (color online).

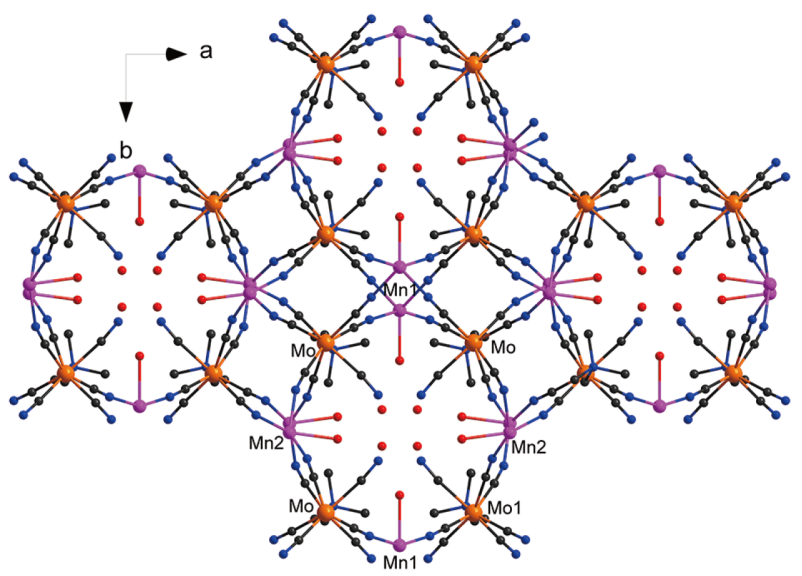

图 $5\left\{\left[\mathrm{~N}\left(\mathrm{CH}_{3}\right)_{4}\right]_{2}\left[\mathrm{Mn}\left(\mathrm{H}_{2} \mathrm{O}\right)\right]_{3}\left[\mathrm{Mo}(\mathrm{CN})_{7}\right]_{2} \cdot 2 \mathrm{H}_{2} \mathrm{O}\right\}_{n}$ 在 $a b$ 面的 晶体结构图. 为了简洁, 省略了氢原子 ${ }^{[46]}$ (网络版彩图)

Figure 5 Crystal structure of the compound $\left\{\left[\mathrm{N}\left(\mathrm{CH}_{3}\right)_{4}\right]_{2}\left[\mathrm{Mn}\left(\mathrm{H}_{2} \mathrm{O}\right)\right]_{3}-\right.$ $\left.\left[\mathrm{Mo}(\mathrm{CN})_{7}\right]_{2} \cdot 2 \mathrm{H}_{2} \mathrm{O}\right\}_{n}$ in the $a b$ plane. Hydrogen atoms have been omitted for clarity [46] (color online)

成了三维框架结构. 在 $b c$ 平面上, 五角双雉的 $\mathrm{Mo}{ }^{\mathrm{III}}$ 中心 分别使用 4 个氧根基团连接Mn2离子，形成波浪形的 $(4,4)$ 网格状的二维层. 这些平行的二维层进一步通过 $\mathrm{Mn} 1(\mathrm{NC})_{4}\left(\mathrm{H}_{2} \mathrm{O}\right)$ 单元连接成 $3 \mathrm{D}$ 框架, 沿着 $c$ 轴方向, 具 有八面体形的一维孔道, 并被配位水分子及结晶水分 子所填充(图5). 由于 $\left[\mathrm{N}\left(\mathrm{CH}_{3}\right)_{4}\right]^{+}$阳离子与 $\mathrm{Mo}^{\mathrm{III}}-\mathrm{Mn}^{\mathrm{II}}$ 阴 离子框架的氢键相互作用, 使得其三维堆积结构更为 紧密, 磁有序温度达到 $86 \mathrm{~K}$.

Larionova等 ${ }^{[47,48]}$ 使用体积更小的 $\mathrm{NH}_{4}{ }^{+}$离子取代 $\left[\mathrm{N}\left(\mathrm{CH}_{3}\right)_{4}\right]^{+}$离子, 合成了两例新的三维化合物, $\left(\mathrm{NH}_{4}\right)_{2}-$ $\left[\mathrm{Mn}_{3}\left(\mathrm{H}_{2} \mathrm{O}\right)_{4}\right]_{3}\left[\mathrm{Mo}(\mathrm{CN})_{7}\right]_{2} \bullet n \mathrm{H}_{2} \mathrm{O}(n=4,5)$. 这两个化合物 具有相似的三维结构, 其在 $b c$ 平面的二维组装与 $\mathrm{K}_{2}$ $\left[\mathrm{Mn}\left(\mathrm{H}_{2} \mathrm{O}\right)_{2}\right]_{3}\left[\mathrm{Mo}(\mathrm{CN})_{7}\right]_{2} \cdot 6 \mathrm{H}_{2} \mathrm{O}$ 化合物极为相似(图6). 由于尺寸较小的 $\mathrm{NH}_{4}{ }^{+}$离子能够嵌入到 $\mathrm{Mo}^{\mathrm{III}}-\mathrm{Mn}^{\mathrm{II}}$ 形成 的立方的空腔中, 使得二维层能够进一步通过 $\mathrm{C} 2 \mathrm{~N} 2$ 氧 根基团连接成三维框架. 磁性测量显示两个化合物的 磁有序温度为 $53 \mathrm{~K}$, 高于包含 $\mathrm{K}^{+}$离子的二维层状化 合物.

这一系列研究表明，通过选用不同的阳离子可以 有效调节 $\mathrm{Mo}^{\mathrm{III}}-\mathrm{Mn}^{\mathrm{II}}$ 网络的维度及拓扑结构, 从而改变 $\mathrm{Mo}^{\mathrm{III}}-\mathrm{Mn}^{\mathrm{II}}$ 分子基磁体的磁有序温度.

\section{3 辅助配体策略构筑 $M{ }^{\mathrm{II}}-\mathrm{Mo}^{\mathrm{III}}{ }^{\text {高 }} \boldsymbol{T}_{\mathrm{c}}$ 磁体}

1999年，Kahn等 ${ }^{[49]}$ 利用五齿配体与 $\mathrm{Mn}^{\mathrm{II}}{ }^{\text {离子制备 }}$ 的前驱体与和 $\left[\mathrm{Mo}(\mathrm{CN})_{7}\right]^{4-}$ 构筑块进行组装, 第一次制 


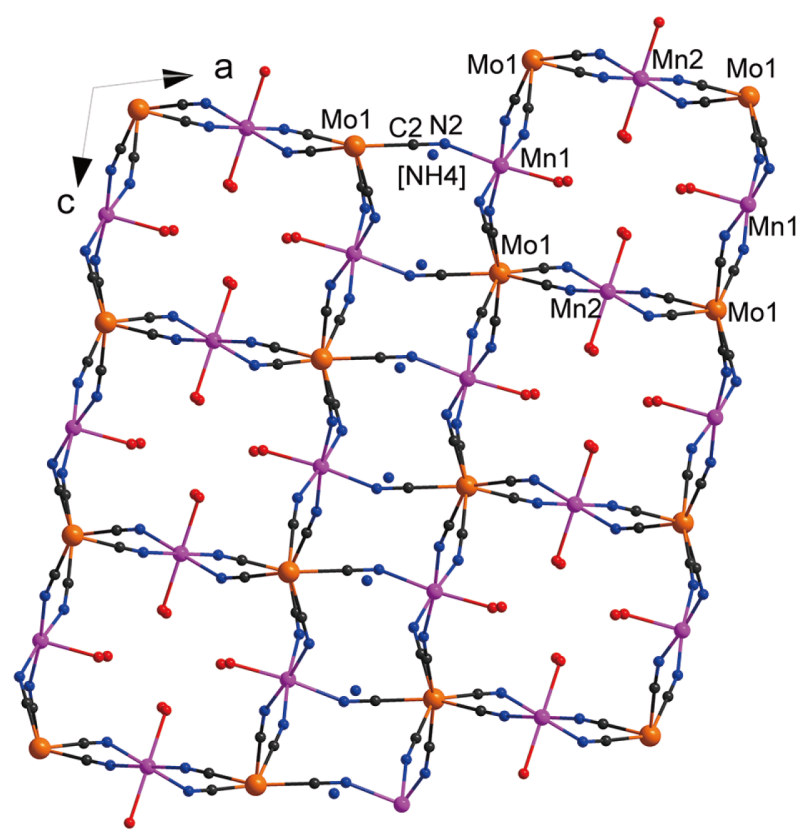

图 $6\left\{\left(\mathrm{NH}_{4}\right)_{2}\left[\mathrm{Mn}_{3}\left(\mathrm{H}_{2} \mathrm{O}\right)_{4}\right]_{3}\left[\mathrm{Mo}(\mathrm{CN})_{7}\right]_{2} \bullet n \mathrm{H}_{2} \mathrm{O}\right\}_{n}$ 在 $a c$ 面的晶 体结构图; 为了简洁, 省略了氢原子 ${ }^{[77,48]}$ (网络版彩图)

Figure 6 Crystal structure of the compound $\left[\left(\mathrm{NH}_{4}\right)_{2}\left[\mathrm{Mn}_{3}\left(\mathrm{H}_{2} \mathrm{O}\right)_{4}\right]_{3}\right.$ $\left.\left[\mathrm{Mo}(\mathrm{CN})_{7}\right]_{2}{ }^{\bullet} n \mathrm{H}_{2} \mathrm{O}\right]_{n}$ in the $a c$ plane. Hydrogen atoms have been omitted for clarity $[47,48]$ (color online).

备了配体螯合的 $\mathrm{Mo}^{\mathrm{III}}-\mathrm{Mn}^{\mathrm{II}}$ 化合物。然而，由于化合物 中部分 $\left[\mathrm{Mo}^{\mathrm{III}}(\mathrm{CN})_{7}\right]^{4-}$ 氧化为 $\left[\mathrm{Mo}^{\mathrm{IV}}(\mathrm{CN})_{8}\right]^{4-}$, 该化合物 仅在 $3 \mathrm{~K}$ 以下具有磁有序行为. 2002 年, 该课题组使用 1,4,7-三氮杂环壬烷(tacn)配体合成了化合物 \{ [ Mn$\left.(\operatorname{tacn})]_{2}\left[\mathrm{Mo}(\mathrm{CN})_{7}\right] \cdot 5 \mathrm{H}_{2} \mathrm{O}\right\}_{n}$, 其磁有序温度可以达到 $90 \mathrm{~K}^{[50]}$. 然而, 由于反应过程极易产生沉淀, 他们并没 有获得该化合物的晶体结构. 尽管如此, 该化合物显示 的高磁有序温度表明该化合物很可能具有三维结构. Sutter课题组 ${ }^{[51]}$ 利用三乙醇胺(tea)贅合配体制备了三 维磁体 $\left\{\left[\mathrm{Mn}_{2} \text { (tea) } \mathrm{Mo}(\mathrm{CN})_{7}\right] \cdot \mathrm{H}_{2} \mathrm{O}\right\}_{n}$. 该化合物结构中分 别包含了一个八面体构型的 $\mathrm{Mn}^{\mathrm{II}}$ 离子和一个四方雉形 的 $\mathrm{Mn}$ 离子. 磁性测量显示该化合物的磁有序温度达 到 $75 \mathrm{~K}$. 另外, 他们通过热处理也获得了无水的化合 物 $\left\{\left[\mathrm{Mn}_{2}(\mathrm{tea}) \mathrm{Mo}(\mathrm{CN})_{7}\right]\right\}_{n}$, 其三维结构与前者相似, 但 是磁有序温度明显高于前者, 达到 $106 \mathrm{~K}$. 这种含水/失 水产物磁性的显著差异(磁海绵现象, magnetic sponge) 主要来源于结构中不同的 $\mathrm{Mn}^{\mathrm{II}}$ 离子的配位构型. 对于 无水化合物, 五配位的 $\mathrm{Mn}^{\mathrm{II}}$ 离子处于四面体配位环境 中, $\mathrm{Mn}-\mathrm{NC}$ 的键长稍短于前者四方锥构型中的 $\mathrm{Mn}-$ $\mathrm{NC}$ 键长, 从而使得 $\mathrm{Mo}^{\mathrm{III}}$ 和 $\mathrm{Mn}^{\mathrm{II}}$ 之间具有更强的磁相互
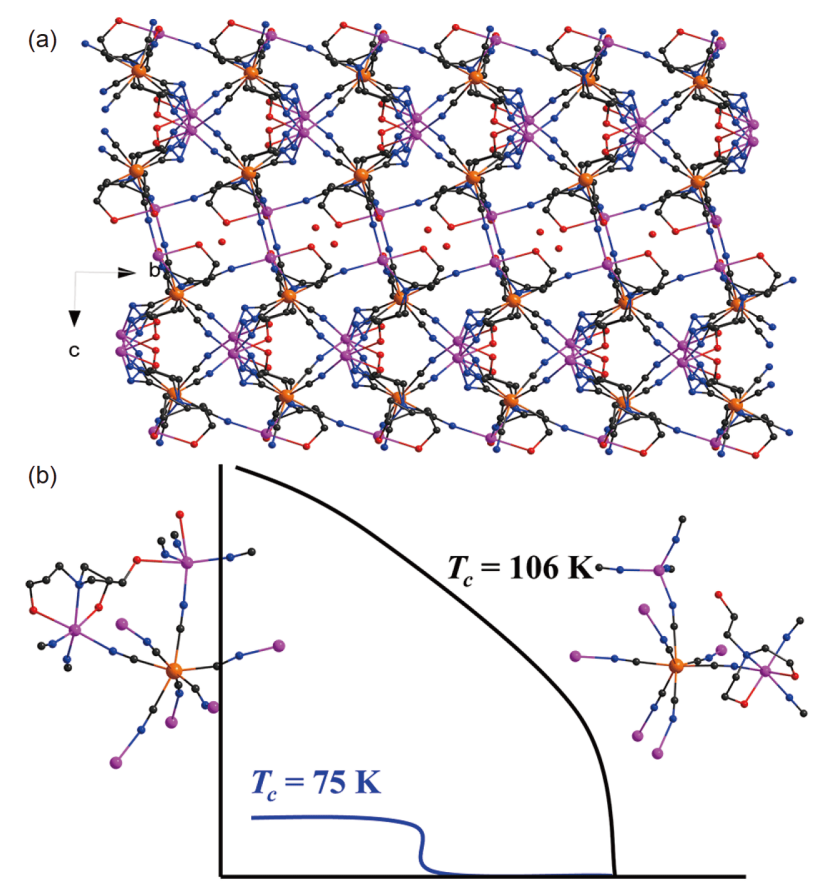

图 7 (a) $\left\{\left[\mathrm{Mn}_{2} \text { (tea) } \mathrm{Mo}(\mathrm{CN})_{7}\right] \cdot \mathrm{H}_{2} \mathrm{O}\right\}_{n}$ 在 $b c$ 面的晶体结构图. 为了简洁, 省略了氢原子; (b) $\left\{\left[\mathrm{Mn}_{2}(\text { tea }) \mathrm{Mo}(\mathrm{CN})_{7}\right] \cdot \mathrm{H}_{2} \mathrm{O}\right\}_{n}$ 和 $\left.\left[\mathrm{Mn}_{2}(\mathrm{tea}) \mathrm{Mo}(\mathrm{CN})_{7}\right]\right\}_{n}$ 化合物的基本单元和磁性质 ${ }^{[1]}$ (网络版 彩图)

Figure 7 (a) Crystal structure of $\left\{\left[\mathrm{Mn}_{2}(\text { tea }) \mathrm{Mo}(\mathrm{CN})_{7}\right] \cdot \mathrm{H}_{2} \mathrm{O}\right\}_{n}$ in the $b c$ plane. Hydrogen atoms have been omitted for clarity; (b) the basic units and magnetic properties of $\left\{\left[\mathrm{Mn}_{2}(\text { tea }) \mathrm{Mo}(\mathrm{CN})_{7}\right] \cdot \mathrm{H}_{2} \mathrm{O}\right\}_{n}$ and $\left\{\left[\mathrm{Mn}_{2}(\text { tea }) \mathrm{Mo}(\mathrm{CN})_{7}\right]\right\}_{n}[51]$ (color online).

作用，这也导致无水化合物具有更高的磁有序温度 (图7).

该课题组也利用手性配体 $N, N$-二甲基丙烯醇制备 了另一例 $\mathrm{Mo}^{\mathrm{III}}-\mathrm{Mn}^{\mathrm{II}}$ 三维磁体, $\left\{\mathrm{Mn}^{\mathrm{II}}\left[\mathrm{Mn}^{\mathrm{II}}(\mathrm{Hdman})\right]_{2}\right.$ $\left.\left[\mathrm{Mo}^{\mathrm{III}}(\mathrm{CN})_{7}\right]_{2} \cdot 2 \mathrm{H}_{2} \mathrm{O}\right\}_{n}$. 该化合物同样具有磁海绵现象: 含水化合物的磁有序温度为 $85 \mathrm{~K}$, 经过脱水处理后, 磁有序温度升高至 $106 \mathrm{~K}^{[52]}$. 此外, Ohkoshi课题组 ${ }^{[53]}$ 和Dunbar课题组 ${ }^{[54,55]}$ 相继开展配体辅助的 $\mathrm{Mo}^{\mathrm{III}}-\mathrm{Mn}^{\mathrm{II}}$ 分子基磁体研究, 并且取得了一系列的研究成果. 这些 研究表明, 引入辅助配体不仅能够改变 $\mathrm{Mn}^{\mathrm{II}}$ 离子的配 位环境, 还可以通过不同类型的有机配体更改 $\mathrm{Mo}^{\mathrm{III}}$ $\mathrm{Mn}^{\mathrm{II}}$ 化合物的拓扑结构, 并进而调节材料的磁学行为.

王新益课题组最早在国内开展 $\left[\mathrm{Mo}(\mathrm{CN})_{7}\right]^{4-}$ 的研 究工作. 2015年, 该课题组将双齿螯合配体引入到 $\mathrm{Mo}^{\mathrm{III}}-\mathrm{Mn}^{\mathrm{II}}$ 体系中, 制备了三例具有相似拓扑结构的 $\mathrm{Mo}^{\mathrm{III}}-\mathrm{Mn}^{\mathrm{II}}$ 三维磁体 ${ }^{[56]}$. 在这三个化合物中, $\mathrm{Mo}^{\mathrm{III}}$ 中心 均处于单帽三棱柱的配位构型, 分别通过 7 个氰根基团 


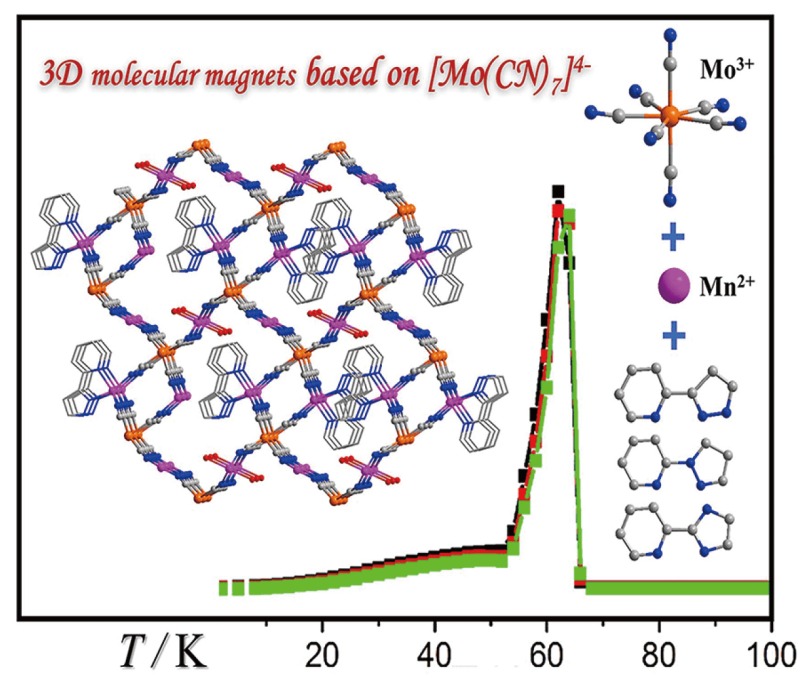

图 8 基于双齿螯合配体的 $\mathrm{Mo}^{\mathrm{III}}-\mathrm{Mn}^{\mathrm{II}}{ }^{\text {三维磁体 }}{ }^{[56]}$ (网络版 彩图)

Figure $8 \mathrm{Mo}^{\mathrm{III}}-\mathrm{Mn}^{\mathrm{II}} 3 \mathrm{D}$ magnets based on the bidentate chelating ligands [56] (color online).

连接 $\mathrm{Mn}^{\mathrm{II}}$ 离子. 由于非常相似的拓扑结构以及金属离 子配位环境, 三个化合物的磁有序温度也非常接近, 分别为 $64 、 66$ 和 $62 \mathrm{~K}$ (图8).

除了这些螯合配体外，该课题组还利用三原子桥 连配体制备了第一例 $\left[\mathrm{Mo}(\mathrm{CN})_{7}\right]^{4-}$ 与甲酸根混合桥连 的三维磁体, $\left\{\left(\mathrm{NH}_{4}\right)_{3}\left[\left(\mathrm{H}_{2} \mathrm{O}\right) \mathrm{Mn}_{3}(\mathrm{HCOO})\right]\left[\mathrm{Mo}(\mathrm{CN})_{7}\right]_{2}\right.$ • $\left.4 \mathrm{H}_{2} \mathrm{O}\right\}_{n}{ }^{[57]}$. 该化合物中包含两个晶体学独立的 $\left[\mathrm{Mo}-(\mathrm{CN})_{7}\right]^{4-}$ 单元和三个 $\mathrm{Mn}^{\mathrm{II}}$ 离子. $\mathrm{Mo}^{\mathrm{III}}$ 中心均处于五 角双雉的配位构型, 并且通过 7 个氧根基团连接 $\mathrm{Mn}^{\mathrm{II}}$ 离 子. 此外, $\mathrm{Mn}$ 吕离子之间分别通过甲酸根以及单原子氧 桥连接. 磁性测量表明其磁有序温度达到 $70 \mathrm{~K}$. 磁滞回 曲线测量显示该化合物的矫顽场达到 $1500 \mathrm{Oe}$, 这也是 当时 $\mathrm{Mo}^{\mathrm{III}}-\mathrm{Mn}^{\mathrm{II}}$ 三维磁体中矫顽力的最大值(图9).

由于该化合物中包含三种不同的桥连模式( $\mathrm{Mo}^{\mathrm{III}}-$ $\mathrm{CN}-\mathrm{Mn}^{\mathrm{II}} 、 \mathrm{Mn}^{\mathrm{II}}-\mathrm{HCOO}-\mathrm{Mn}^{\mathrm{II}}$ 和 $\mathrm{Mn}^{\mathrm{II}}-\mathrm{O}-\mathrm{Mn}^{\mathrm{II}}$ ), 其三维结 构中存在两种不同的“蝴蝶型”拓扑结构. 在这些结构 中, 自旋中心竞争的反铁磁相互作用可能会导致化合 物内存在自旋阻挫以及磁矩的非线性排列. 这种自旋 阻挫现象以及磁矩的非线性排列也在他们组最近的两 例 $\mathrm{Mo}^{\mathrm{III}}-\mathrm{Mn}^{\mathrm{II}}$ 三维磁体中观察到 ${ }^{[58]}$. 在这两个化合物中, $\mathrm{DMF}$ 作为终端配体与 $\mathrm{Mn}^{\mathrm{II}}$ 离子配位, 而 $\mathrm{DEF}$ 作为桥连 配体连接两个 $\mathrm{Mn}^{\mathrm{II}}$ 离子. $\mathrm{Mo}^{\mathrm{III}}$ 中心均处于单帽三棱柱 配位构型, 自旋中心之间分别通过 $\mathrm{CN}$ 和 $\mu_{2}-\mathrm{O}$ 桥连接. 两个化合物的磁有序温度达到 $80 \mathrm{~K}$ 以上. 另外, 两个

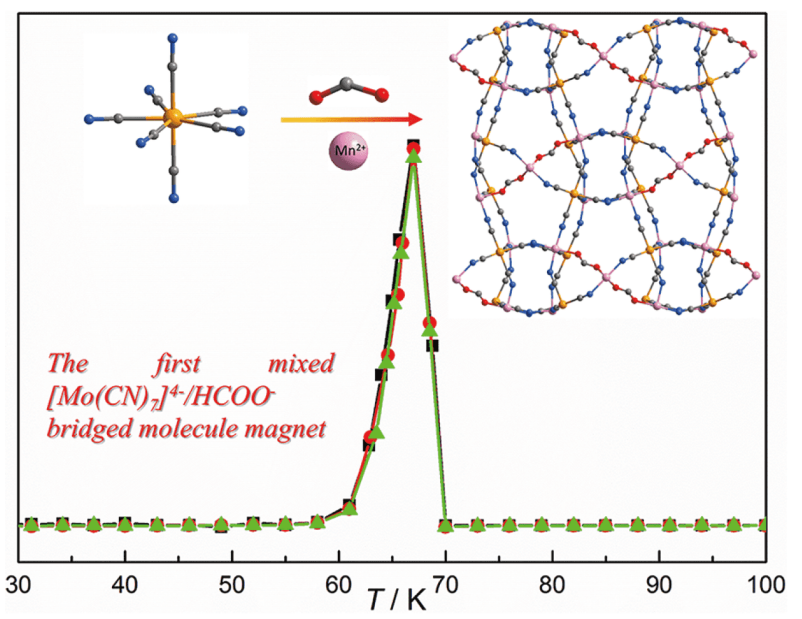

图 $9\left[\mathrm{Mo}(\mathrm{CN})_{7}\right]^{4-}$ 与 $\mathrm{HCOO}^{-}$混合桥连的三维磁体 ${ }^{[57]}$ (网络 版彩图)

Figure 9 Mixed $\left[\mathrm{Mo}(\mathrm{CN})_{7}\right]^{4-}$ and $\mathrm{HCOO}^{-}$bridged 3D magnet [57] (color online).

化合物的交流磁化率曲线和场冷-零场冷曲线在 $45 \mathrm{~K}$ 附近出现较小的峰, 这种现象与 $\alpha$ 相和 $\beta$ 相相似, 可能是 源于低温下的自旋重排.

值得注意的是，尽管这两个化合物的磁有序温度 较高, 但是其矫顽场仅为 $200 \mathrm{Oe}$. 而且, 早期报道的高 $T_{\mathrm{c}} \mathrm{Mo}^{\mathrm{III}}-\mathrm{Mn}^{\mathrm{II}}$ 三维磁体的矫顽场均较小. 这种情况可能 与 $\mathrm{Mo}{ }^{\mathrm{III}}$ 中心的配位构型密切相关. 理论研究表明, 五角 双雉的 $\left[\mathrm{Mo}(\mathrm{CN})_{7}\right]^{4-}$ 具有很强的磁各向异性, 而且 $\mathrm{Mo}^{\mathrm{III}}-\mathrm{Mn}^{\mathrm{II}}$ 之间的磁交换也具有各向异性. Pinkowicz课 题组 ${ }^{[59]}$ 在最近报道的四个 $\mathrm{Mo}^{\mathrm{III}}-\mathrm{Mn}^{\mathrm{II}}$ 三维化合物中探 讨了 $\mathrm{Mo}^{\mathrm{III}}-\mathrm{Mn}^{\mathrm{II}}$ 三维磁体的磁各向异性与 $\mathrm{Mo}^{\mathrm{III}}$ 中心配 位构型之间的关系. 研究结果显示, 五角双雉的 $\mathrm{Mo}^{\mathrm{III}}$ 中 心对获得大的矫顽场非常有利. 此外, $\mathrm{Mo}^{\mathrm{III}}-\mathrm{CN}-\mathrm{Mn}^{\mathrm{II}}$ 的 连接方式以及 C-N-Mn弯曲的角度也与矫顽场的大小 密切相关.

\section{4 其他 $\mathrm{Mo}^{\mathrm{III}}-\mathrm{M}^{\mathrm{II}}{ }^{\text {高 }} \boldsymbol{T}_{\mathrm{c}}$ 磁体}

截至目前, 绝大多数基于 $\left[\mathrm{Mo}(\mathrm{CN})_{7}\right]^{4-}$ 的磁有序化 合物都是 $\mathrm{Mo}^{\mathrm{III}}-\mathrm{Mn}^{\mathrm{II}}$ 化合物, 通过引入阳离子和辅助配 体调控其结构及磁性. 然而, 通过使用 $\mathrm{Fe}^{\mathrm{II}} 、 \mathrm{Co}^{\mathrm{II}}$ 、 $\mathrm{Ni}^{\mathrm{II}} 、 \mathrm{~V}^{\mathrm{II} / \mathrm{II}}$ 和 $\mathrm{Cr}^{\mathrm{II} / \mathrm{III}}$ 等磁各向异性金属离子取代 $\mathrm{Mn}^{\mathrm{II}}$ 离 子，有望显著调节自旋中心之间的磁耦合，进而调节 $\left[\mathrm{Mo}(\mathrm{CN})_{7}\right]^{4-}$ 化合物的磁有序温度. 然而, 合成上的困 难在很大程度上限制了这类化合物的发展. 尽管在 2000 年Kahn等 ${ }^{[60]}$ 就报道了一例 $\mathrm{Fe}^{\mathrm{II}}-\mathrm{Mo}^{\mathrm{III}}$ 三维磁体, 其 
有序温度为 $65 \mathrm{~K}$. 然而, 由于没有获得该化合物的晶体 结构, 无法进行详细的磁结构相关的分析. 直到2016 年, 王新益课题组 ${ }^{[61]}$ 才报道了具有单晶结构的 $\mathrm{Mo}^{\mathrm{III}}-\mathrm{Fe}^{\mathrm{II}}$ 三维磁体: $\left\{\mathrm{Fe}_{2}\left(\mathrm{H}_{2} \mathrm{O}\right)_{5}\left[\mathrm{Mo}(\mathrm{CN})_{7}\right] \cdot 5 \mathrm{H}_{2} \mathrm{O}\right\}_{n}$ 和 $\left\{\left[\mathrm{NH}_{2}\left(\mathrm{CH}_{3}\right)_{2}\right]_{2} \mathrm{Fe}_{5}\left(\mathrm{H}_{2} \mathrm{O}\right)_{10}\left[\mathrm{Mo}(\mathrm{CN})_{7}\right]_{3} \cdot 8 \mathrm{H}_{2} \mathrm{O}\right\}_{n}$. 两个化 合物具有不同的三维拓扑结构, 磁性测量显示它们的 磁有序温度均为 $65 \mathrm{~K}$, 略高于早期报道的 $\mathrm{Mo}^{\mathrm{III}}-\mathrm{Mn}^{\mathrm{II}}$ 三 维磁体的磁有序温度. 这些结果表明 $\mathrm{Fe}^{\mathrm{II}}-\mathrm{Mo}^{\mathrm{III}}$ 之间的 磁耦合可能比 $\mathrm{Mo}^{\mathrm{III}}-\mathrm{Mn}^{\mathrm{II}}$ 要强, 这有利于制备高 $T_{\mathrm{c}}$ 分子 基磁体. 另外, 这两个化合物在低温下均能观察到明 显的磁滞回曲线, 矫顽场分别达到 6000 和 1900 Oe. 该 研究成果为开展 $\mathrm{Fe}^{\mathrm{II}}$ 分子基磁体研究提供了思路 (图10).

除了 $\mathrm{Fe}^{\mathrm{II}}-\left[\mathrm{Mo}(\mathrm{CN})_{7}\right]^{4-}$ 三维磁体外, Ohkoshi课题 组 ${ }^{[62]}$ 在 2009 年报道了 $\mathrm{Ni}^{\mathrm{II}}-\left[\mathrm{Mo}(\mathrm{CN})_{7}\right]^{4-}$ 三维磁体: $\left\{\mathrm{K}_{0.6} \mathrm{Ni}_{1.7}\left[\mathrm{Mo}(\mathrm{CN})_{7}\right] \cdot 5 \mathrm{H}_{2} \mathrm{O} \bullet 0.5 \mathrm{CH}_{3} \mathrm{OH}\right\}_{n}$. 尽管并没有获 得晶体产物, 但是他们通过化合物的粉末衍射图谱确 定了其结构组成. 在结构中, $\mathrm{Mo}^{\mathrm{III}}$ 中心处于单帽三棱 柱配位构型, 并且通过 7 个氰根基团与 $\mathrm{Ni}^{\mathrm{II}}$ 离子配位. 磁测量表明 $\mathrm{Mo}^{\mathrm{III}}$ 和 $\mathrm{Ni}^{\mathrm{II}}$ 之间通过氯根传递铁磁相互作 用, 其自发磁化的有序温度约为 $28 \mathrm{~K}$.

利用相似的合成方法, Ohkoshi课题组 ${ }^{[53]}$ 在2010年 又报道了 $\mathrm{V}^{\mathrm{II}}-\left[\mathrm{Mo}(\mathrm{CN})_{7}\right]^{4-}$ 三维磁体: $\left\{\mathrm{V}^{\mathrm{II}}{ }_{2}\left[\mathrm{Mo}{ }^{\mathrm{III}}(\mathrm{CN})_{7}\right]\right.$ • (pyrimidine $\left.)_{2} \cdot 2 \mathrm{H}_{2} \mathrm{O}\right\}_{n}$. 尽管没有获得该化合物的单晶, 但通过对比XRD图谱以及元素分析表征, 他们认为其 结构和相似的 $\mathrm{Mn}^{\mathrm{II}}-\left[\mathrm{Mo}(\mathrm{CN})_{7}\right]^{4-}$ 化合物同构(图11), 结

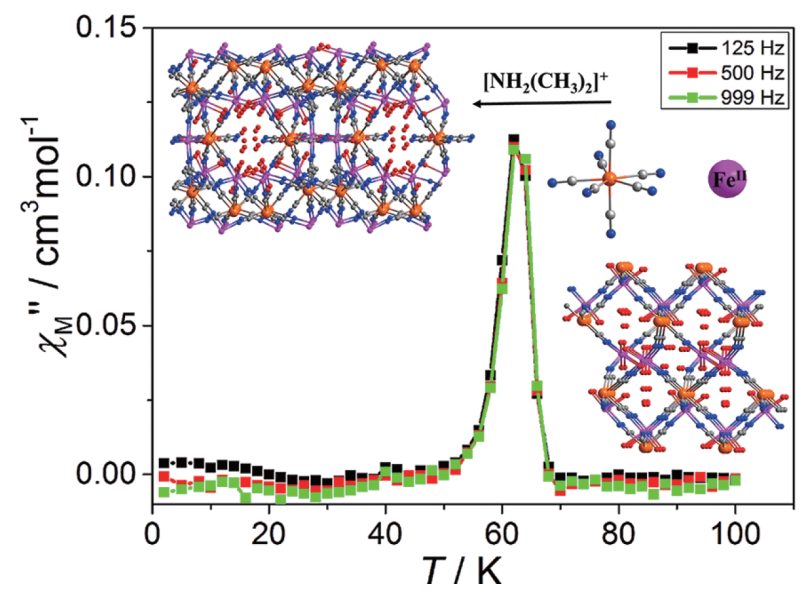

图 $10 \mathrm{Fe}^{\mathrm{II}}-\left[\mathrm{Mo}(\mathrm{CN})_{7}\right]^{4-}$ 三维磁体的晶体结构和磁性 ${ }^{[61]}$ (网 络版彩图)

Figure 10 Crystal structures and magnetic properties of $\mathrm{Fe}^{\mathrm{II}}-[\mathrm{Mo}$ $\left.(\mathrm{CN})_{7}\right]^{4-}$ 3D magnets $[61]$ (color online).

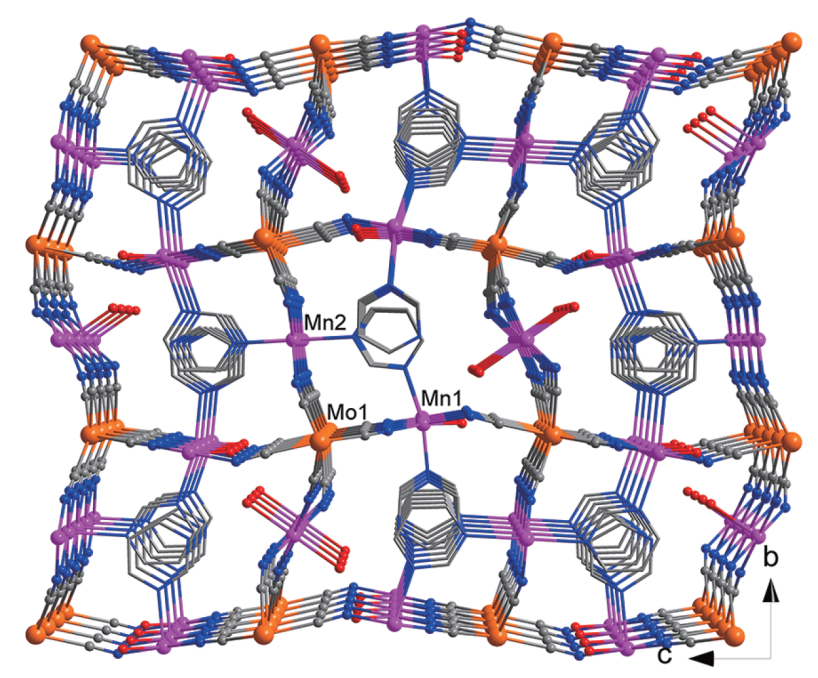

图 $\left.11\left\{\mathrm{Mn}_{2}^{\mathrm{II}}\left[\mathrm{Mo}^{\mathrm{III}}(\mathrm{CN})_{7}\right] \cdot \text { (pyrimidine }\right)_{2} \cdot 2 \mathrm{H}_{2} \mathrm{O}\right\}_{n}$ 在 $b c$ 面的晶 体结构图. 为了简洁, 省略了氢原子 ${ }^{[53]}$ (网络版彩图)

Figure 11 Crystal structure of compound $\left\{\mathrm{Mn}_{2}{ }_{2}\left[\mathrm{Mo}^{\mathrm{III}}(\mathrm{CN})_{7}\right] \cdot\right.$ (pyrimidine $\left.)_{2} \cdot 2 \mathrm{H}_{2} \mathrm{O}\right\}_{n}$ in the $b c$ plane. Hydrogen atoms have been omitted for clarity [53] (color online).

构中同时含有嘧啶分子作为桥连配体. 磁性测量显示, $\mathrm{Mn}^{\mathrm{II}}$ 化合物的居里温度仅有 $47 \mathrm{~K}$, 而 $\mathrm{V}^{\mathrm{II}}$ 的同构化合物 的居里温度达到 $110 \mathrm{~K}$, 是基于 $\left[\mathrm{Mo}(\mathrm{CN})_{7}\right]^{4-}$ 的分子基 磁体中的最高值. 如此高的磁有序温度主要源于强的 $\mathrm{V}^{\mathrm{II}}$ 和 $\mathrm{Mo}^{\mathrm{III}}$ 之间的磁交换作用. 此外, 该化合物的矫顽 场也远高于同构的 $\mathrm{Mn}^{\mathrm{II}}-\left[\mathrm{Mo}(\mathrm{CN})_{7}\right]^{4-}$ 化合物. 这些结果 表明, $\mathrm{V}^{\mathrm{II}}-\left[\mathrm{Mo}(\mathrm{CN})_{7}\right]^{4-}$ 体系对于构筑高磁有序温度的 分子基磁体具有很大的潜力.

\section{4 基于 $\left[\mathrm{Mo}(\mathrm{CN})_{7}\right]^{4-}$ 的低维磁体}

由于 $\left[\mathrm{Mo}(\mathrm{CN})_{7}\right]^{4-}$ 的二维或三维化合物具有高的 磁有序温度及磁各向异性, 其磁各向异性的来源吸引 了研究人员的关注. 2003年, Mironov等 ${ }^{[35]}$ 使用动力学 交换理论模型分析了四个典型的 $\mathrm{Mo}^{\mathrm{III}}-\mathrm{Mn}^{\mathrm{II}}$ 三维磁体. 由于 $\mathrm{Mo}^{\mathrm{III}}$ 离子的轨道简并性和强的旋轨耦合, 其未被 淬灭的轨道角动量导致 $\left[\mathrm{Mo}(\mathrm{CN})_{7}\right]^{4-}$ 基态具有很高的 $g$ 因子各向异性. 另外, 理论研究指出, $\mathrm{Mo}^{\mathrm{III}}-\mathrm{CN}-\mathrm{Mn}^{\mathrm{II}}$ 本 身的磁交换作用也具有很强的磁各向异性. 基于此, $\mathrm{Mo}^{\mathrm{III}}-\mathrm{Mn}^{\mathrm{II}}$ 之间的自旋耦合甚至无法通过铁磁或反铁 磁相互作用准确描述, 也无法使用经典的海森堡自旋 哈密顿表示. 研究指出, 对于具有理想的五角双雉配 位几何的 $\left[\mathrm{Mo}(\mathrm{CN})_{7}\right]^{4-}$ 中心, 其轴向配位的 $\mathrm{Mo}^{\mathrm{III}}-\mathrm{CN}-$ 
$\mathrm{Mn}$ 连接之间的磁交换作用可以通过Ising型自旋哈密 顿 $H=-J S_{\mathrm{Mo}}^{2} S_{\mathrm{Mn}}^{2}$ 来描述, 而赤道位置的 $\mathrm{Mo}^{\mathrm{III}}-\mathrm{CN}-\mathrm{Mn}^{\mathrm{II}}$ 之间的磁交换则可以用自旋哈密顿 $H=-J_{\mathrm{xy}}\left(S^{x}{ }_{\mathrm{Mo}} S^{x}{ }_{\mathrm{Mn}}{ }^{+}\right.$ $\left.S_{\mathrm{Mo}}^{\nu} S_{\mathrm{Mn}}^{\nu}\right)-J_{\mathrm{z}}\left(S_{\mathrm{Mo}}^{\mathrm{Z}} S_{\mathrm{Mn}}^{\mathrm{Z}}\right)$ 来描述. 其中, Ising型各向异性 磁交换是构筑高能垒单分子磁体的重要来源. 理论研 究表明, 简单的由轴向 $\mathrm{CN}$ 基团连接的双核 $\mathrm{Mo}^{\mathrm{III}}-\mathrm{Mn}^{\mathrm{II}}$ 化合物即可能表现出单分子磁体行为. 这是一个非常 有效的构筑高能垒单分子磁体的研究策略. 基于这一 理论指导, 研究人员开始将目光转向 $\left[\mathrm{Mo}(\mathrm{CN})_{7}\right]^{4-}$ 的低 维分子磁体.

\section{1 基于 $\left[\mathrm{Mo}(\mathrm{CN})_{7}\right]^{4-}$ 的零维分子簇}

尽管理论预测认为可以通过构筑基于 $\left[\mathrm{Mo}(\mathrm{CN})_{7}\right]^{4-}$ 的零维团簇来构筑高能垒单分子磁体, 但 在实验上, 基于 $\left[\mathrm{Mo}(\mathrm{CN})_{7}\right]^{4-}$ 的零维团簇却非常难以合 成, 一直没有实现. 这主要是由于 $\left[\mathrm{Mo}(\mathrm{CN})_{7}\right]^{4-}$ 较多的负 电荷以及7 个氧根基团极易生成高维分子团簇. 通过多 齿鳌合配体，占据 $3 \mathrm{~d}$ 金属的配位点，有望合成基于 $\left[\mathrm{Mo}(\mathrm{CN})_{7}\right]^{4-}$ 的低维化合物. 2010年, Dunbar课题组 ${ }^{[63]}$ 制备了由 $\left[\mathrm{Mo}(\mathrm{CN})_{7}\right]^{4-}$ 桥连的分子簇合物 $\left[\mathrm{Mo}_{8} \mathrm{Mn}_{14}\right]$ : $\left[\mathrm{Mn}(\mathrm{dpop})\left(\mathrm{H}_{2} \mathrm{O}\right)_{2}\right]_{2}\left[\left\{\mathrm{Mo}(\mathrm{CN})_{7}\right\}_{8}\{\mathrm{Mn}(\mathrm{dpop})\}_{10}\{\mathrm{Mn}-\right.$ $\left.\left.(\mathrm{dpop})\left(\mathrm{H}_{2} \mathrm{O}\right)\right\}_{4}\right] \cdot x \mathrm{H}_{2} \mathrm{O}$. 该化合物由 8 个 $\left[\mathrm{Mo}(\mathrm{CN})_{7}\right]^{4-}$ 单元 通过轴向和赤道平面氧根基团连接 14 个 $[\mathrm{Mn}(\mathrm{dpop})]^{2+}$ 部分形成独特的 22 核阴离子簇, 另有两个 $[\mathrm{Mn}(\mathrm{dpop})]^{2+}$ 部分作为抗衡阳离子(图12). 所有的 $\mathrm{Mo}^{\mathrm{III}}$ 中心均处于 畸变的五角双雉配位环境中. 化合物的直流磁化率曲 线和等温磁化强度曲线表明 $\mathrm{Mo}^{\mathrm{III}}-\mathrm{CN}-\mathrm{Mn}^{\mathrm{II}}$ 之间为反铁 磁相互作用. $\mathrm{Mo}^{\mathrm{III}}$ 和 $\mathrm{Mn}^{\mathrm{II}}{ }^{\mathrm{G}}$ 旋反向排列使得该分子团 簇具有 $S=31$ 的自旋基态，这是当时氧基化合物中最高 的自旋基态值. 另外, 化合物在 $1.8 \mathrm{~K}$ 温度下可以观察 到明显的磁滞回线, 矫顽场为 $180 \mathrm{Oe}$, 剩余磁化强度为 $18.5 \mu_{\mathrm{B}}$. 尽管化合物的交流磁化率有明显的频率依赖 行为, 但是其实部磁化率位移参数仅为 0.035 , 处于自 旋玻璃的取值范围内，表明该分子簇并非单分子磁体， 而表现出自旋玻璃的行为.

除了 $\left[\mathrm{Mo}_{8} \mathrm{Mn}_{14}\right]$ 分子簇以外, Dunbar课题组 ${ }^{[64]}$ 最近 也报道了一例 $\left[\mathrm{Mo}_{6} \mathrm{Ni}_{12}\right]$ 轮状簇合物. 该化合物与之前 报道的 $\left[\mathrm{Mo}^{\mathrm{IV}}(\mathrm{CN})_{8}\right]_{6}[\mathrm{Ni}(\mathrm{dtpd})]_{12}\left(\mathrm{H}_{2} \mathrm{O}\right)_{6}$ 化合物类似 ${ }^{[65]}$, 由 6 个 $\left[\mathrm{Mo}(\mathrm{CN})_{7}\right]^{4-}$ 基团连接 12 个 $[\mathrm{Ni}(\mathrm{dtpd})]^{2+}$ 部分组成, 所有的 $\mathrm{Mo}{ }^{\mathrm{III}}$ 中心均处于单帽三棱柱配位环境中(图13). 磁性测量表明, $\mathrm{Mo}^{\mathrm{III}}$ 和 $\mathrm{Ni}^{\mathrm{II}}$ 之间为铁磁相互作用, 并且

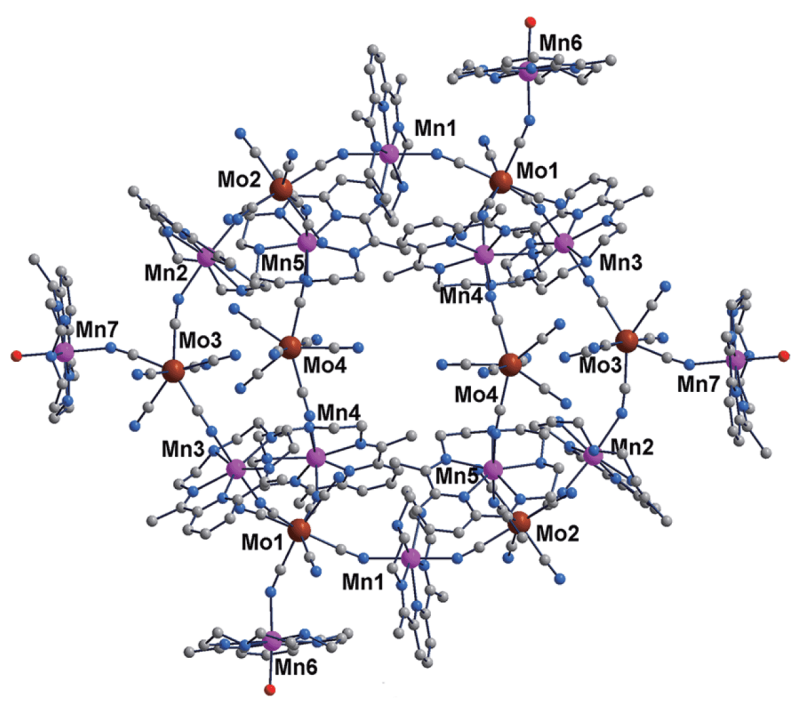

图 $12 \mathrm{Mo}_{8} \mathrm{Mn}_{14}$ 分子簇的晶体结构图. 为了简洁, 省略了氢 原子 ${ }^{[63]}$ (网络版彩图)

Figure 12 Crystal structure of $\mathrm{Mo}_{8} \mathrm{Mn}_{14}$ cluster. Hydrogen atoms have been omitted for clarity [63] (color online).

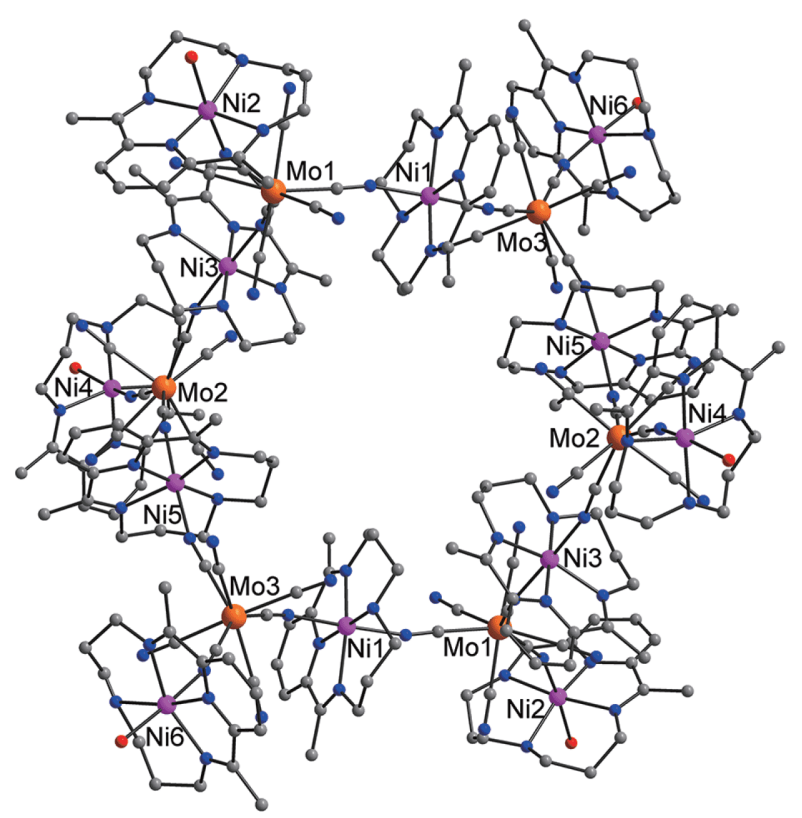

图 $13 \mathrm{Mo}_{6} \mathrm{Ni}_{12}$ 分子簇的晶体结构图. 为了简洁, 省略了氢 原子 ${ }^{[64]}$ (网络版彩图)

Figure 13 Crystal structure of $\mathrm{Mo}_{6} \mathrm{Ni}_{12}$ cluster. Hydrogen atoms have been omitted for clarity [64] (color online).

具有 $S=15$ 的自旋基态. 尽管 $\left[\mathrm{Mo}_{8} \mathrm{Mn}_{14}\right]$ 和 $\left[\mathrm{Mo}_{6} \mathrm{Ni}_{12}\right]$ 分 子簇都具有很大的自旋基态，但是它们都不是单分子 磁体. 这种情况一方面是由于高核的分子簇削弱了化 合物的磁各向异性，另一方面可能是由于 $\mathrm{Mo}^{\mathrm{III}}$ 和 $3 \mathrm{~d}$ 金 
属离子之间的磁耦合并非Ising型磁耦合.

为了获得 $\left[\mathrm{Mo}(\mathrm{CN})_{7}\right]^{4-}$ 桥连的单分子磁体, 强的Ising 型的 $\mathrm{Mo}^{\mathrm{III}}-\mathrm{Mn}^{\mathrm{II}}$ 各向异性磁交换至关重要. 这要求 $\left[\mathrm{Mo}(\mathrm{CN})_{7}\right]^{4-}$ 尽可能处于规则的五角双雉配位几何中, 而且采取轴向桥连模式连接其他金属中心. 2013年, 王 新益课题组 ${ }^{[66]}$ 报道了基于 $\left[\mathrm{Mo}(\mathrm{CN})_{7}\right]^{4-}$ 桥连的单分子 磁体: $\left[\mathrm{Mn}\left(\mathrm{L}_{\mathrm{N}_{5} \mathrm{Me}}\right) \mathrm{H}_{2} \mathrm{O}\right]_{2}\left[\mathrm{Mo}(\mathrm{CN})_{7}\right] \cdot 6 \mathrm{H}_{2} \mathrm{O}$. 单晶研究显 示, $\mathrm{Mo}{ }^{\mathrm{III}}$ 中心处于轻微畸变的五角双雉配位环境中, 并且使用两个轴向氰根基团连接 $\mathrm{Mn}^{\mathrm{II}}$ 离子, 形成三核 $\left[\mathrm{Mn}_{2} \mathrm{Mo}\right]$ 簇合物 (图 14). 磁性测量表明, 该化合物在 $3.2 \mathrm{~K}$ 以下具有单分子磁体行为, 其自旋翻转能垒达到 $58 \mathrm{~K}$. 另外, 该化合物在1.8 3.0 K温度范围内具有非常 明显的源于量子隧穿的台阶状磁滞回线. 尽管 $\mathrm{Mo}^{\mathrm{III}}$ 和 $\mathrm{Mn}^{\mathrm{II}}$ 之间的反铁磁相互作用导致化合物仅仅只有 $S=$ 9/2的自旋基态, 但是其自旋翻转能垒是当时氰基桥连 单分子磁体中的最大值, 几乎可以和基态为 10 的 $\left[\mathrm{Mn}_{12}\right]$ 单分子磁体相謧美. 由于低自旋的 $\mathrm{Mo}^{\mathrm{III}}(S=1 / 2)$ 和 $\mathrm{Mn}^{\mathrm{II}}$ 离子具有非常小的零场分裂能, 该单分子磁体 的磁各向异性主要源于 $\mathrm{Mo}^{\mathrm{III}}-\mathrm{Mn}^{\mathrm{II}}$ 之间的各向异性磁 交换.

同时，他们也报道了两例赤道平面氧根配位的 $\left[\mathrm{Mn}_{2} \mathrm{Mo}\right]$ 三核化合物. 磁性测量显示它们只表现为简 单的顺磁行为, 进一步表明轴向连接模式有利于获得 强的Ising型自旋耦合. 这些研究结果从实验上证明了 理论研究工作者提出的模型, 说明了各向异性磁交换
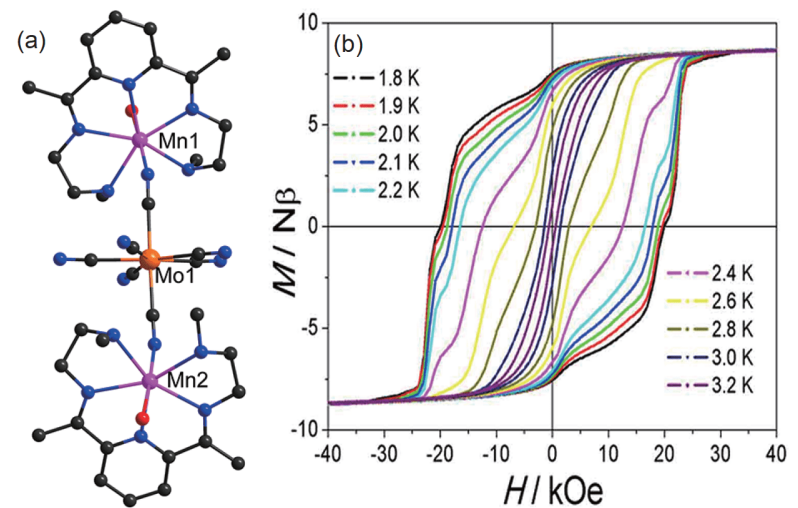

图 $14\left[\mathrm{Mn}\left(\mathrm{L}_{\mathrm{N}_{5} \mathrm{Me}}\right) \mathrm{H}_{2} \mathrm{O}\right]_{2}\left[\mathrm{Mo}(\mathrm{CN})_{7}\right] \cdot 6 \mathrm{H}_{2} \mathrm{O}$ 三核簇的晶体结构 (a)以及在1.8 3.2 K下的磁滞回曲线(b). 为了简洁, 省略了氢 原子 ${ }^{[6]}$ (网络版彩图)

Figure 14 Crystal structure of $\left[\mathrm{Mn}\left(\mathrm{L}_{\mathrm{N}_{5} \mathrm{Me}}\right) \mathrm{H}_{2} \mathrm{O}\right]_{2}\left[\mathrm{Mo}(\mathrm{CN})_{7}\right] \cdot 6 \mathrm{H}_{2} \mathrm{O}$ trinuclear cluster (a) and hysteresis loops at 1.8-3.2 K (b). Hydrogen atoms have been omitted for clarity [66] (color online).
在构筑高能垒单分子磁体中的重要作用, 为构筑更多 高能垒单分子磁体提供了新的思路.

\section{2 基于 $\left[\mathrm{Mo}(\mathrm{CN})_{7}\right]^{4-}$ 的一维链}

$\left[\mathrm{Mn}_{2} \mathrm{Mo}\right]$ 单分子磁体的合成不仅证实了理论研究 的结果, 同时也为理论工作者提供了实际模型, 尤其是 不同的桥连模式表现出不同的磁学性质吸引研究人员 对其单分子磁体行为的探讨. Mironov ${ }^{[67]}$ 进一步通过 理论计算指出, $\left[\mathrm{Mn}_{2} \mathrm{Mo}\right.$ ]三核化合物的自旋哈密顿可 以通过 $H_{\mathrm{eff}}=-J_{\mathrm{xy}}\left(S_{\mathrm{Mo}^{x}}^{x} S_{\mathrm{Mn}}^{x}+S^{y}{ }_{\mathrm{Mo}} S_{\mathrm{Mn}}^{y}\right)-J_{z}\left(S_{\mathrm{Mo}}^{z} S_{\mathrm{Mn}}^{z}\right)$ 进行 描述. 它们的单分子磁体行为的差异主要取决于各向 异性交换参数 $J_{z}$ 和 $J_{x y}$ 的相对大小. 对于轴向配位的 $\left[\mathrm{Mn}_{2} \mathrm{Mo}\right]$ 化合物, 强的Ising型各向异性自旋耦合 $\left(J_{z}=\right.$ $\left.-34 \mathrm{~cm}^{-1}, J_{x y}=-11 \mathrm{~cm}^{-1}\right)$ 能产生双势阱自旋能级, 导致 化合物表现出单分子磁体行为. 相反, 两个赤道平面配 位的 $\left[\mathrm{Mn}_{2} \mathrm{Mo}\right.$ 化合物中具有 $x y$ 型磁交换, 由于 $\left|J_{z}\right|<\left|J_{x y}\right|$, 两个化合物均具有单势阱的自旋能级裂分, 从而表现 为简单的顺磁性. 此外, 研究也指出, 通过增加化合物 的轴向 $\mathrm{Mo}^{\mathrm{III}}-\mathrm{CN}-\mathrm{Mn}^{\mathrm{II}}$ 连接数有助于进一步提高其自旋 翻转能垒. 基于这一理论, 研究者有望通过构筑一维 $\mathrm{Mn}^{\mathrm{II}}-\left[\mathrm{Mo}(\mathrm{CN})_{7}\right]^{4-}$ 链状化合物来构筑高能垒单链磁体.

然而, 实验上, 基于 $\left[\mathrm{Mo}(\mathrm{CN})_{7}\right]^{4-}$ 的链状化合物很 少被报道. 2016年，王新益课题组 ${ }^{[68]}$ 报道了基于 $[\mathrm{Mo}-$ $\left.(\mathrm{CN})_{7}\right]^{4-}$ 的一维磁链化合物: $\left\{\left[\mathrm{Mn}\left(\mathrm{L}_{\mathrm{N}_{5} \mathrm{C}_{10}}\right)\right]_{2}\left[\mathrm{Mo}(\mathrm{CN})_{7}\right]\right.$ $\left.\cdot 2 \mathrm{H}_{2} \mathrm{O}\right\}_{n}$. 在该化合物中, $\mathrm{Mo}{ }^{\mathrm{III}}$ 中心处于畸变的五角双 锥配位环境中, 分别使用两个赤道平面的氰根基团和 一个轴向氰根基团与三个 $\mathrm{Mn}^{\mathrm{II}}$ 离子配位(图15(a)), 通 过赤道平面1,3-位置的氰根基团连接，化合物沿 $b$ 方向 形成一维单“之”字型链状结构. 磁性测量显示该化合 物在 $5.6 \mathrm{~K}$ 以下具有长程磁有序行为. 尽管交流磁化率 曲线的实部和虚部均有频率依赖现象, 但是其位移参 数(Mydosh参数)处于自旋玻璃数值范围内, 表明该一 维链并非单链磁体. 其磁有序可能源于其中链与链之 间通过大量的氢键相互作用所传递的磁作用. 另外, 化合物中的有机配体具有非常严重的位置无序, 这导 致了该化合物中的自旋玻璃行为.

2017年，王庆伦等 ${ }^{[69]}$ 也报道了类似的 $\mathrm{Mn}^{\mathrm{II}}$-[Mo$\left.(\mathrm{CN})_{7}\right]^{4-}$ 一维链, $\left\{[\mathrm{Mn}(\mathrm{dpop})]\left[\mathrm{Mn}(\mathrm{dpop})\left(\mathrm{H}_{2} \mathrm{O}\right)\right][\mathrm{Mo}-\right.$ $\left.\left.(\mathrm{CN})_{7}\right] \cdot 7.5 \mathrm{H}_{2} \mathrm{O}\right\}_{n}$. 其中, 五角双雉的 $\mathrm{Mo}^{\mathrm{III}}$ 中心分别通 过两个赤道平面氰根基团和一个轴向氰根基团连接 $\mathrm{Mn}^{\mathrm{II}}$ 离子. 尽管该化合物具有单链磁体的特征, 但由于 


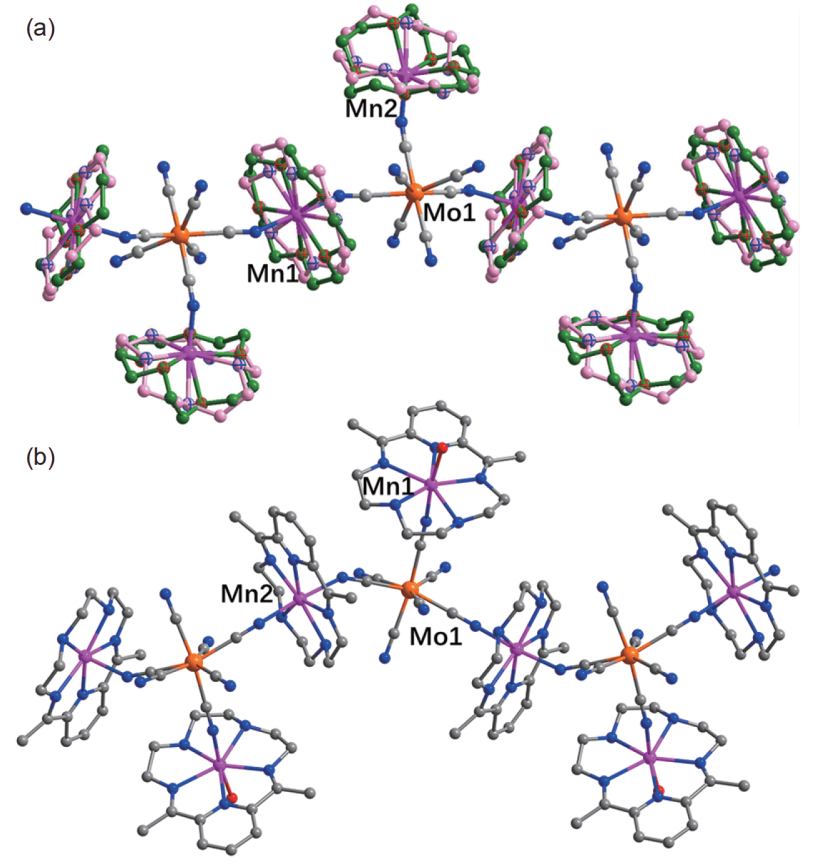

图 15 (a) $\left.\left\{\left[\mathrm{Mn}\left(\mathrm{L}_{\mathrm{N}_{5} \mathrm{C}_{10}}\right)\right]_{2}\right\}\left[\mathrm{Mo}(\mathrm{CN})_{7}\right] \cdot 2 \mathrm{H}_{2} \mathrm{O}\right\}_{n}$ 的一维链状结 构 ${ }^{[68]}$; (b) $\left\{\left[\mathrm{Mn}(\text { dpop) }]\left[\mathrm{Mn}(\mathrm{dpop})\left(\mathrm{H}_{2} \mathrm{O}\right)\right]\left[\mathrm{Mo}(\mathrm{CN})_{7}\right] \cdot 7.5 \mathrm{H}_{2} \mathrm{O}\right\}_{n}\right.$ 的一维链状结构 ${ }^{[69]}$. 为了简洁, 省略了氢原子 ${ }^{[68,69]}$ (网络版 彩图)

Figure 15 (a) 1D chain structures of $\left\{\left[\mathrm{Mn}\left(\mathrm{L}_{\mathrm{N}_{5} \mathrm{C}_{10}}\right)\right]_{2}\right\}\left[\mathrm{Mo}(\mathrm{CN})_{7}\right]$ - $\left.2 \mathrm{H}_{2} \mathrm{O}\right\}_{n}$ [68]; (b) 1D chain structures of $\{[\mathrm{Mn}(\mathrm{dpop})][\mathrm{Mn}(\mathrm{dpop})-$ $\left.\left.\left(\mathrm{H}_{2} \mathrm{O}\right)\right]\left[\mathrm{Mo}(\mathrm{CN})_{7}\right] \cdot 7.5 \mathrm{H}_{2} \mathrm{O}\right\}_{n}$ [69]. Hydrogen atoms have been omitted for clarity $[68,69]$ (color online).

该化合物内较少的轴向 $\mathrm{Mo}^{\mathrm{III}}-\mathrm{CN}-\mathrm{Mn}^{\mathrm{II}}$ 连接以及不可忽 略的链间磁相互作用导致其自旋翻转能垒仅为 $69.5 \mathrm{~K}$.

为了获得高能垒单链磁体, 不仅要求 $\mathrm{Mo}{ }^{\mathrm{III}}$ 中心处 于五角双雉配位构型, 还要尽可能增加轴向 $\mathrm{Mo}^{\mathrm{III}}-\mathrm{CN}-$ $M n$ 连接的数量. 此外, 可忽略的链间磁相互作用也非 常重要. 最近, 王新益课题组 ${ }^{[70]}$ 通过配体设计, 合成了 一例新颖的 $\mathrm{Mn}^{\mathrm{II}}-\mathrm{Mo}^{\mathrm{III}}$ 单链磁体: $\left\{\left[\mathrm{Mn}(\text { bida })\left(\mathrm{H}_{2} \mathrm{O}\right)\right]_{2}-\right.$ $\left.\left[\mathrm{Mo}(\mathrm{CN})_{7}\right] \cdot 6 \mathrm{H}_{2} \mathrm{O}\right\}_{n}$. 该化合物的基本单元由 $\mathrm{Mn}_{2} \mathrm{Mo}$ 组 成, 三价的 $\mathrm{Mo}^{\mathrm{III}}$ 中心处于五角双雉的配位环境中. 与 之前的两例一维链不同，该链中的 $\mathrm{Mo}^{\mathrm{III}}$ 中心分别使用 两个轴向氰根基团连接两个 $\mathrm{Mn}^{\mathrm{II}}$ 离子. 这种连接模式 与之前报道的 $\mathrm{Mn}_{2} \mathrm{Mo}$ 单分子磁体极为相似，表明链内 能够产生很强的各向异性磁交换. 因此, 一维的链状 结构可以看作由 $\mathrm{Mn}_{2} \mathrm{Mo}$ 单分子磁体通过赤道平面氧 根基团连接而成(图16). 磁性测量表明该化合物为单 链磁体, 并且具有非常大的畴壁能, $\Delta_{\xi}=57 \mathrm{~K}$. 其自旋 翻转能垒则达到 $178 \mathrm{~K}$, 高于大部分单链磁体的弛豫能 垒. 更为显著的是, 该化合物在 $5.2 \mathrm{~K}$ 以下呈现出源于

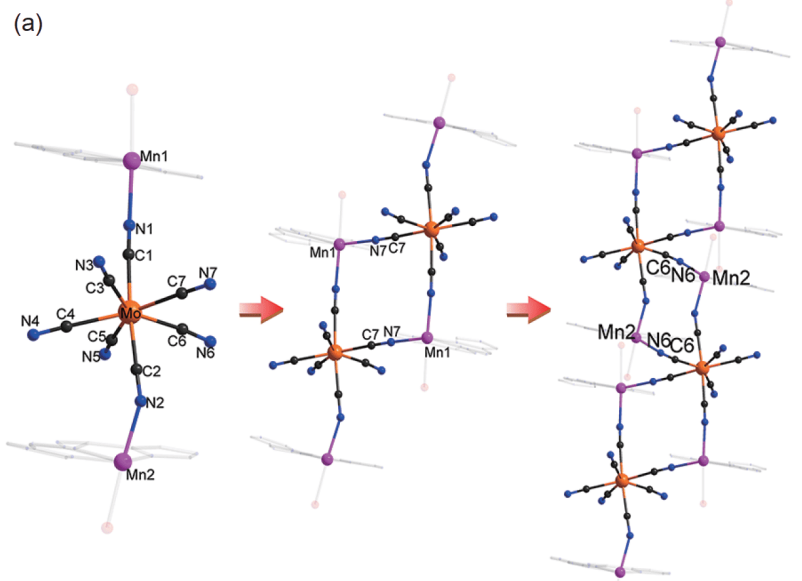

(b)

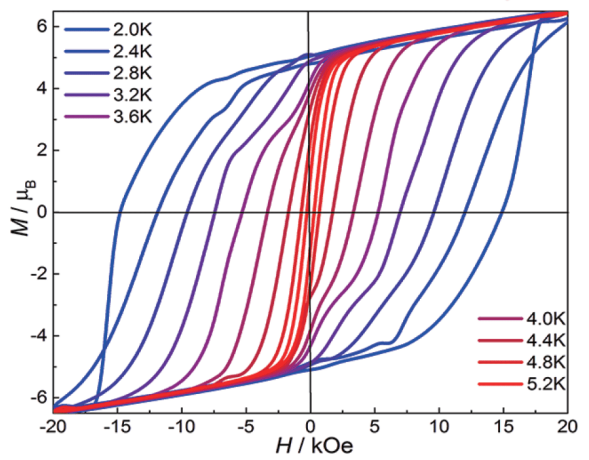

图 16 (a) $\left\{\left[\mathrm{Mn} \text { (bida) }\left(\mathrm{H}_{2} \mathrm{O}\right)\right]_{2}\left[\mathrm{Mo}(\mathrm{CN})_{7}\right] \cdot 6 \mathrm{H}_{2} \mathrm{O}\right\}_{n}$ 的逐步组装 结构图: 从三核到六核再到 $1 \mathrm{D}$ 链. 为了简洁, 省略了氢原子; (b) 取向单晶在不同温度下的磁滞回曲线 ${ }^{[70]}$ (网络版彩图)

Figure 16 (a) Step-wise view of the structure of $\left\{\left[\mathrm{Mn}(\text { bida })\left(\mathrm{H}_{2} \mathrm{O}\right)\right]_{2}-\right.$ $\left.\left[\mathrm{Mo}(\mathrm{CN})_{7}\right] \cdot 6 \mathrm{H}_{2} \mathrm{O}\right\}_{n}$ : from trinuclear to hexanuclear to the 1D chain. Hydrogen atoms have been omitted for clarity. (b) Magnetic hysteresis loops of oriented single crystals at different temperatures [70] (color online).

量子隧穿的台阶状磁滞回线. 在 $2.0 \mathrm{~K}$ 时, 该单链磁体 的矫顽场达到 15000 Oe.

同样, 与之前报道的 $\left[\mathrm{Mn}_{2} \mathrm{Mo}\right]$ 单分子磁体相似, 该 单链磁体的磁各向异性主要源于 $\mathrm{Mo}^{\mathrm{III}}-\mathrm{Mn}^{\mathrm{II}}$ 之间的各 向异性磁交换. 因此, 该单链磁体的有效哈密顿既不能 通过Ising模型也不能通过海森堡模型进行描述, 与其 他单链磁体存在明显的差异. 另外, 该化合物的自旋 翻转能垒高于大部分单链磁体, 表明各向异性磁交换 在构筑高能垒单链磁体领域也具有很大的潜力.

\section{5 基于 $\left[\mathrm{Mo}(\mathrm{CN})_{7}\right]^{4-}$ 的客体调控磁转换材料}

磁转换材料是近年来分子磁性领域的前沿和热 点, 受到研究人员的普遍关注. 这类材料的磁学性质能 
够通过外界刺激(如光照、温度、压力、电场、磁 场、客体分子等)进行调节 ${ }^{[71]}$. 其中, 客体分子导致的 磁转换行为在分子磁性材料领域被广泛报道. 化合物 中的客体分子在一定条件下的脱附和吸附，或和其他 客体分子之间的可逆交换，可以导致化合物的磁学参 数, 如自旋交叉温度、磁有序温度和磁弛豫参数等发 生相应的变化 ${ }^{[72 \sim 7]}$. 此外, 在一定条件下, 客体分子的 得失和交换还能引发固相化学反应，使金属中心发生 配位键重组, 进而导致结构和磁性的巨大变化 ${ }^{[75]}$.

自从第一例基于 $\left[\mathrm{Mo}(\mathrm{CN})_{7}\right]^{4-}$ 的分子磁性材料被 报道以来，研究人员便一直关注客体分子对其结构和 磁学性质的影响. 如最早的 $\alpha$ 相和 $\beta$ 相 $\mathrm{Mo}^{\mathrm{III}}-\mathrm{Mn}^{\mathrm{II}}$ 三维磁 体，在经过脱水处理后其磁有序温度从 $51 \mathrm{~K}$ 增加至 $65 \mathrm{~K}$. 而且， $\alpha$ 相经过热处理之后可以在 $5 \mathrm{~K}$ 以下具有 $0.85 \mathrm{kOe}$ 的矫顽场 ${ }^{[39]}$. 此外, 二维的 $\left\{\mathrm{K}_{2}\left[\mathrm{Mn}\left(\mathrm{H}_{2} \mathrm{O}\right)_{2}\right]_{3}\right.$ $\left.\left[\mathrm{Mo}(\mathrm{CN})_{7}\right]_{2} \cdot 6 \mathrm{H}_{2} \mathrm{O}\right\}_{n}$ 化合物也显示出客体分子敏感的 磁有序行为, 该化合物在真空下部分脱水后, 其磁有序 温度从 $39 \mathrm{~K}$ 增加至 $72 \mathrm{~K}$, 并且具有 $1.3 \mathrm{kOe}$ 的矫顽场 ${ }^{[44]}$

2007 年, Sutter等 ${ }^{[52]}$ 合成了一例具有多孔结构的 $\mathrm{Mo}^{\mathrm{III}}-\mathrm{Mn}^{\mathrm{II}}$ 三维分子磁体, $\mathrm{Mn}^{\mathrm{II}}\left[\mathrm{Mn}^{\mathrm{II}}(\mathrm{Hdman})\right]_{2}\left[\mathrm{Mo}^{\mathrm{III}}\right.$ $\left.(\mathrm{CN})_{7}\right]_{2} \cdot 2 \mathrm{H}_{2} \mathrm{O}$. 在该化合物中, $\mathrm{Mo}^{\mathrm{III}}$ 中心处于单帽三棱 柱配位构型，使用7个氧根基团连接 $\mathrm{Mn}^{\mathrm{II}}$ 离子形成柔性 的多孔框架结构, 客体水分子分布于沿着 $c$ 轴方向形成 的一维孔道内(图17(a)). 磁性测量显示该三维磁体的 磁有序温度达到 $85 \mathrm{~K}$, 而脱水处理后其磁有序温度能 够达到106 K (图17(b)). 有趣的是, 该化合物可以重新 吸收水分子回到最初的结构. 热分析和变温XRD研究 证实了该化合物的脱水和重吸收水的过程是完全可 逆的.

Sutter课题组 ${ }^{[76]}$ 也报道了一例具有 4 种磁性转换的 三维 $\mathrm{Mo}^{\mathrm{III}}-\mathrm{Mn}^{\mathrm{II}}$ 亚铁磁体, $\left\{\left[\mathrm{K}_{2}\left(\mathrm{H}_{2} \mathrm{O}\right)_{4} \mathrm{Mn}_{5}\left(\mathrm{H}_{2} \mathrm{O}\right)_{8}-\right.\right.$ $\left.\left.(\mathrm{MeCN})\left\{\mathrm{Mo}(\mathrm{CN})_{7}\right\}_{3}\right] \cdot 2 \mathrm{H}_{2} \mathrm{O}\right\}_{n}$ (图18(a)). 在该化合物 中, $\mathrm{Mo}^{\mathrm{III}}$ 中心连接 7 个 $\mathrm{Mn}^{\mathrm{II}}$ 离子形成三维金属有机框 架，并且沿着 $a$ 方向具有 $18 \times 22 \AA$ 的一维孔道. $\mathrm{K}^{+}$离子 和客体分子填充在孔道内(图18(a))。该化合物具有较 高的热稳定性, 并且显示出可逆的客体分子交换行为. 在不同的条件下, 化合物的磁有序温度可在61 82 K之 间转变, 而矫顽场在70 1770 Oe之间变化(图18(b)).

此外, Dunbar课题组 ${ }^{[54]}$ 也获得了基于 $\left[\mathrm{Mo}(\mathrm{CN})_{7}\right]^{4-}$ 的客体调控磁转换材料. 利用五角双雉的 $\mathrm{Mn}^{\mathrm{II}}$ 前驱体 与 $\left[\mathrm{Mo}(\mathrm{CN})_{7}\right]^{4-}$ 自组装, 她们获得了一例三维化合物:
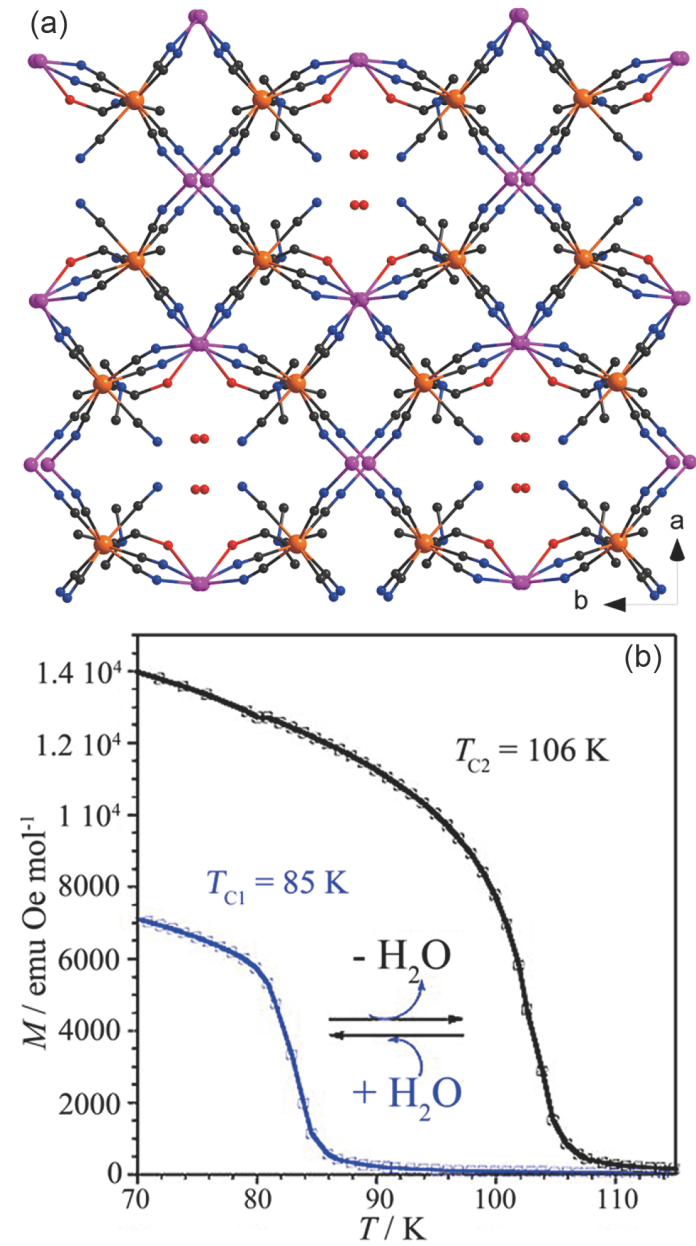

图 17 (a) $\left\{\mathrm{Mn}^{\mathrm{II}}\left[\mathrm{Mn}^{\mathrm{II}}(\mathrm{Hdman})\right]_{2}\left[\mathrm{Mo}^{\mathrm{III}}(\mathrm{CN})_{7}\right]_{2} \cdot 2 \mathrm{H}_{2} \mathrm{O}\right\}_{n}$ 在 $a b$ 面 的晶体结构图. 为了简洁, 省略了氢原子; (b) 脱水和吸水材 料的 $M-T$ 曲线显示临界温度的变化 ${ }^{[52]}$ (网络版彩图)

Figure 17 (a) Crystal structure of $\left\{\mathrm{Mn}^{\mathrm{II}}\left[\mathrm{Mn}^{\mathrm{II}}(\mathrm{Hdman})\right]_{2}\left[\mathrm{Mo}^{\mathrm{III}}(\mathrm{CN})_{7}\right]_{2}\right.$ $\left.\cdot 2 \mathrm{H}_{2} \mathrm{O}\right\}_{n}$ in the $a b$ plane. Hydrogen atoms have been omitted for clarity; (b) the $M-T$ curves of the dehydrated and rehydrated materials indicating the reversible changes in the critical temperatures [52] (color online).

$\left\{[\mathrm{Mn}(\mathrm{dpop})]_{3}\left[\mathrm{Mn}(\mathrm{dpop})\left(\mathrm{H}_{2} \mathrm{O}\right)\right]\left[\mathrm{Mo}(\mathrm{CN})_{7}\right]_{2}\right\}_{n}$. 该化合物 的基本单元由两个 $\left[\mathrm{Mo}(\mathrm{CN})_{7}\right]^{4-}$ 连接一个 $[\mathrm{Mn}(\mathrm{dpop}-$ $\left.\left(\mathrm{H}_{2} \mathrm{O}\right)\right]^{2+}$ 和三个 $[\mathrm{Mn}(\mathrm{dpop})]^{2+}$ 部分组成. $\mathrm{Mo}^{\mathrm{III}}$ 中心均处 于五角双雉的配位环境中, Mo1分别通过两个赤道平 面氰根基团和两个轴向氰根基团连接四个 $\mathrm{Mn}^{\mathrm{II}}$ 离子, 而 $\mathrm{Mo} 2$ 赤道平面有三个 $\mathrm{CN}$ 与 $\mathrm{Mn}^{\mathrm{II}}$ 离子配位, 轴向位置 有一个 $\mathrm{CN}$ 与 $\mathrm{Mn}^{\mathrm{II}}$ 离子配位(图19(a)). 三维框架结构高 度有序, 其拓扑结构为 $6.10^{2} .10^{2}$. 结构中. 沿着 $c$ 轴方向 存在被客体水分子占据的一维孔道. 该化合物在 $2.6 \mathrm{~K}$ 以下表现为磁有序并有一定的自旋玻璃行为. $1.8 \mathrm{~K}$, 
(a)
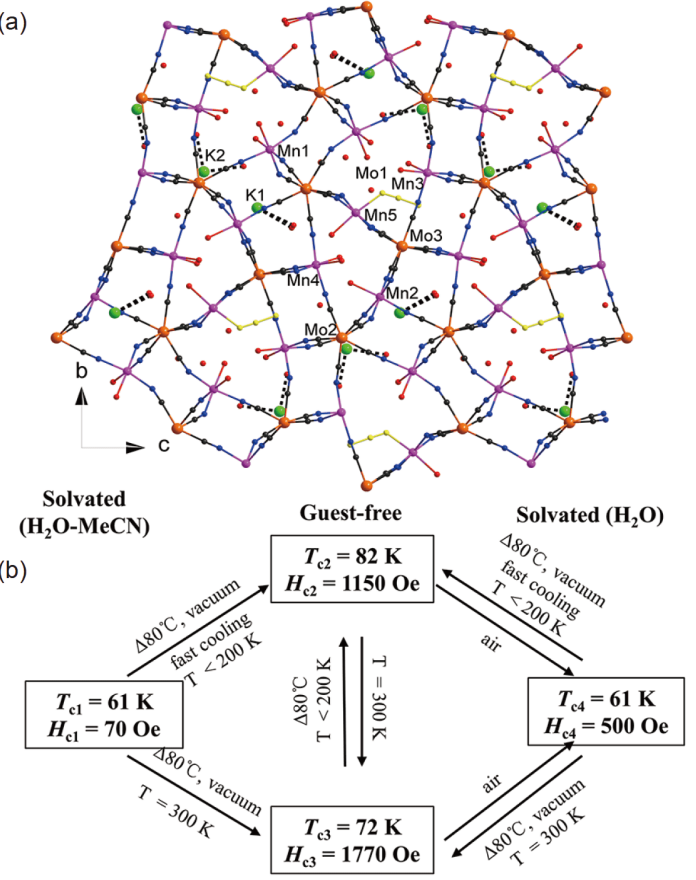

图 18 (a) $\left\{\left[\mathrm{K}_{2}\left(\mathrm{H}_{2} \mathrm{O}\right)_{4} \mathrm{Mn}_{5}\left(\mathrm{H}_{2} \mathrm{O}\right)_{8}(\mathrm{MeCN})\left\{\mathrm{Mo}(\mathrm{CN})_{7}\right\}_{3}\right]\right.$ $\left.\cdot 2 \mathrm{H}_{2} \mathrm{O}\right\}_{n}$ 在 $b c$ 面的晶体结构图. 为了简洁, 省略了氢原子; $(\mathrm{b})$ 用于磁性选择性转换的条件 ${ }^{[76]}$ (网络版彩图)

Figure 18 (a) Crystal structure of $\left\{\left[\mathrm{K}_{2}\left(\mathrm{H}_{2} \mathrm{O}\right)_{4} \mathrm{Mn}_{5}\left(\mathrm{H}_{2} \mathrm{O}\right)_{8}(\mathrm{MeCN})-\right.\right.$ $\left.\left.\left\{\mathrm{Mo}(\mathrm{CN})_{7}\right\}_{3}\right] \cdot 2 \mathrm{H}_{2} \mathrm{O}\right\}_{n}$ in the $b c$ plane. Hydrogen atoms have been omitted for clarity; (b) conditions to be applied for selective conversion of the magnetic attributes [76] (color online).

可以观察到较小的磁滞回曲线, 矫顽场约为 $90 \mathrm{Oe}$.

有趣的是，该化合物在 $125^{\circ} \mathrm{C}$ 下热处理 $8 \mathrm{~h}$ 后，[Mn$\left(\mathrm{dpop}\left(\mathrm{H}_{2} \mathrm{O}\right)\right]^{2+}$ 部分配位的水分子进一步被 $\left[\mathrm{Mo}(\mathrm{CN})_{7}\right]^{4-}$ 取代, 转变为新的三维结构—— $\left\{[\mathrm{Mn}(\mathrm{dpop})]_{2}[\mathrm{Mo}-\right.$ $\left.\left.(\mathrm{CN})_{7}\right] \cdot 2 \mathrm{H}_{2} \mathrm{O}\right\}_{n}$ (图19(c)). 其基本单元由 $\left\{[\mathrm{Mn}(\mathrm{dpop})]_{2}-\right.$ $\left.\left[\mathrm{Mo}(\mathrm{CN})_{7}\right]_{2}\right\}$ 部分和两个水分子组成(图19(b)), 在转换 过程中, $\left[\mathrm{Mo}(\mathrm{CN})_{7}\right]^{4-}$ 的桥连模式发生极大的变化, $\mathrm{Mo}$ 吕中心分别使用两个轴向氧根基团和两个赤道平面 氧根基团连接 4 个 $[\mathrm{Mn}(\mathrm{dpop})]^{2+}$ 部分, 形成 $6^{2} .6^{2} .6^{2} .6^{2} .8^{2} .8^{2}$ 的拓扑结构. 由于结构的改变, 其磁学 性质也明显不同，新的三维化合物具有 $20 \mathrm{~K}$ 的磁有序 温度，而矫顽场达到305 Oe. 显著增加的磁有序温度 主要由于新的化合物中具有更多的 $\mathrm{Mo}^{\mathrm{III}}-\mathrm{CN}-\mathrm{Mn}^{\mathrm{II}}{ }^{\mathrm{I}}$ 磁交 换通道。

除了三维磁体的客体调控磁转换行为外, 王新益 课题组 ${ }^{[77]}$ 也在低维分子磁体中通过客体分子实现磁 性的可逆转换. 他们使用席夫碱配体bimda与 $\mathrm{Mn}^{\mathrm{II}}$ 及 $\left[\mathrm{Mo}^{\mathrm{III}}(\mathrm{CN})_{7}\right]^{4-}$ 进行组装, 制备了一例新的 $\mathrm{Mn}_{2} \mathrm{Mo}$ 三核
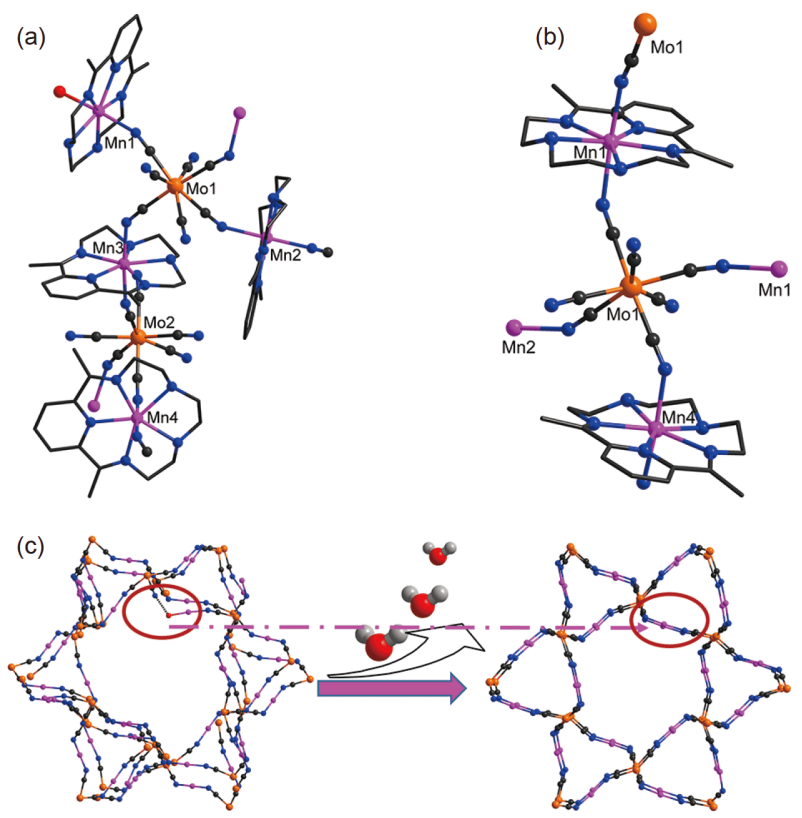

图 19 (a) $\left\{[\mathrm{Mn}(\mathrm{dpop})]_{3}\left[\mathrm{Mn}(\mathrm{dpop})\left(\mathrm{H}_{2} \mathrm{O}\right)\right]\left[\mathrm{Mo}(\mathrm{CN})_{7}\right]_{2}\right\}_{n}$ 的基 本结构单元; (b) $\left\{[\mathrm{Mn}(\mathrm{dpop})]_{2}\left[\mathrm{Mo}(\mathrm{CN})_{7}\right] \cdot 2 \mathrm{H}_{2} \mathrm{O}\right\}_{n}$ 的基本结构 单元; (c) 从 $\left\{[\mathrm{Mn}(\mathrm{dpop})]_{3}\left[\mathrm{Mn}(\mathrm{dpop})\left(\mathrm{H}_{2} \mathrm{O}\right)\right]\left[\mathrm{Mo}(\mathrm{CN})_{7}\right]_{2}\right\}_{n}$ 到 $\left\{[\mathrm{Mn}(\mathrm{dpop})]_{2}\left[\mathrm{Mo}(\mathrm{CN})_{7}\right] \cdot 2 \mathrm{H}_{2} \mathrm{O}\right\}_{n}$ 的单晶转变 ${ }^{[54]}$ (网络版彩图) Figure 19 (a) The structural unit of $\left\{[\mathrm{Mn}(\mathrm{dpop})]_{3}\left[\mathrm{Mn}(\mathrm{dpop})\left(\mathrm{H}_{2} \mathrm{O}\right)\right]\right.$ $\left.\left[\mathrm{Mo}(\mathrm{CN})_{7}\right]_{2}\right\}_{n}$; (b) the structural unit of $\left\{[\mathrm{Mn}(\mathrm{dpop})]_{2}\left[\mathrm{Mo}(\mathrm{CN})_{7}\right]\right.$ $\left.-2 \mathrm{H}_{2} \mathrm{O}\right\}_{n}$; (c) crystal-to-crystal transformation from $\left\{[\mathrm{Mn}(\mathrm{dpop})]_{3}[\mathrm{Mn}-\right.$ (dpop) $\left.\left.\left(\mathrm{H}_{2} \mathrm{O}\right)\right]\left[\mathrm{Mo}(\mathrm{CN})_{7}\right]_{2}\right\}_{n}$ to $\left\{[\mathrm{Mn}(\mathrm{dpop})]_{2}\left[\mathrm{Mo}(\mathrm{CN})_{7}\right] \cdot 2 \mathrm{H}_{2} \mathrm{O}\right\}_{n}[54]$ (color online).
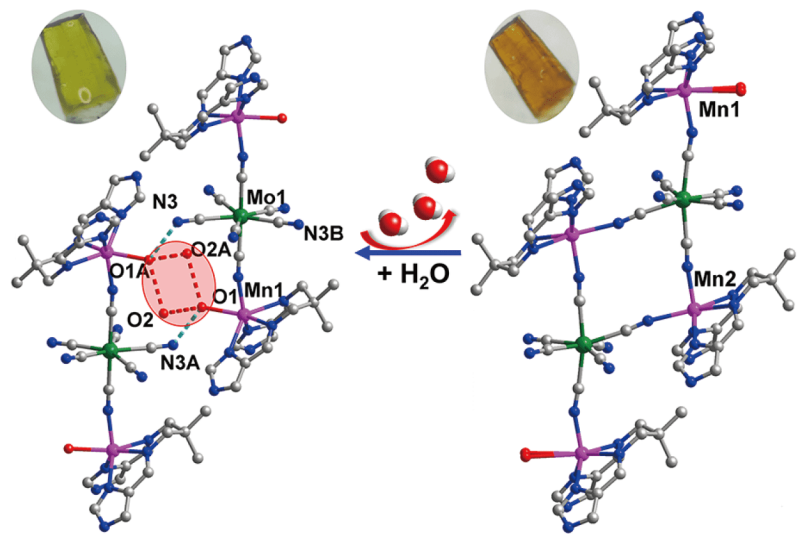

图 20 从 $\left[\mathrm{Mn}(\text { bimda })\left(\mathrm{H}_{2} \mathrm{O}\right)\right]_{2}\left[\mathrm{Mo}(\mathrm{CN})_{7}\right] \cdot 2 \mathrm{H}_{2} \mathrm{O}$ 到 $[\mathrm{Mn}($ bimda)$\left.\left(\mathrm{H}_{2} \mathrm{O}\right)\right]_{2}[\mathrm{Mn}(\mathrm{bimda})]_{2}\left[\mathrm{Mo}(\mathrm{CN})_{7}\right]_{2}$ 的单晶转变 ${ }^{[77]}$ (网络版彩图) Figure 20 Crystal-to-crystal transformation from [Mn(bimda)$\left.\left(\mathrm{H}_{2} \mathrm{O}\right)\right]_{2}\left[\mathrm{Mo}(\mathrm{CN})_{7}\right] \cdot 2 \mathrm{H}_{2} \mathrm{O}$ to $\left[\mathrm{Mn}(\text { bimda })\left(\mathrm{H}_{2} \mathrm{O}\right)\right]_{2}[\mathrm{Mn}(\text { bimda })]_{2}[\mathrm{Mo}-$ $\left.(\mathrm{CN})_{7}\right]_{2}[77]$ (color online).

团簇: $\left[\mathrm{Mn}(\text { bimda })\left(\mathrm{H}_{2} \mathrm{O}\right)\right]_{2}\left[\mathrm{Mo}(\mathrm{CN})_{7}\right] \cdot 2 \mathrm{H}_{2} \mathrm{O}$ (图20). 单 晶研究显示, $\mathrm{Mo}^{\mathrm{III}}$ 中心处于畸变的五角双雉配位构型, 并且使用两个轴向的氰根基团桥连 $\mathrm{Mn}^{\mathrm{II}}{ }^{\mathrm{⿱亠凶}}$ 子。磁性测 
量表明, 该化合物在低温下表现为单分子磁体. 有趣的 是, 该化合物在真空加热条件下可以通过单晶-单晶转 变发生配位水分子的失去以及新的 $\mathrm{Mn}-\mathrm{N}$ 键的形成, 从 $\left[\mathrm{Mn}_{2} \mathrm{Mo}\right]$ 三核分子团簇转变成 $\left[\mathrm{Mn}_{4} \mathrm{Mo}_{2}\right]$ 六核分子团 簇 $\left[\mathrm{Mn}(\mathrm{L})\left(\mathrm{H}_{2} \mathrm{O}\right)\right]_{2}[\mathrm{Mn}(\mathrm{L})]_{2}\left[\mathrm{Mo}(\mathrm{CN})_{7}\right]_{2}$. 有意思的是, 在 六核分子簇中, $\mathrm{Mo}{ }^{\mathrm{III}}$ 中心不再具有五角双雉配位构型, 这使得新的六核化合物失去了单分子磁体行为. 而且, 该单晶转变过程是完全可逆的: 六核分子簇合物吸收 空气中的水分子可以重新转变为三核结构. 在这个体 系中，实现了单晶状态下单分子磁体的开关调控以及 各向异性磁交换作用的可逆转换.

\section{6 总结与展望}

本文综述了基于 $\left[\mathrm{Mo}(\mathrm{CN})_{7}\right]^{4-}$ 的分子磁性材料的 研究进展. 与八面体构型的 $\left[\mathrm{M}(\mathrm{CN})_{6}\right]^{3-}$ 构筑块以及八 配位的 $\left[\mathrm{M}(\mathrm{CN})_{8}\right]^{3-}$ 相比，七配位的 $\mathrm{Mo}^{\mathrm{III}}$ 中心能够和其 他金属之间产生强的磁交换并具有更强的磁各向异 性, 这使得 $\left[\mathrm{Mo}(\mathrm{CN})_{7}\right]^{4-}$ 在构筑高 $T_{\mathrm{c}}$ 分子磁体和分子纳 米磁体等方面尤为重要. 目前, 大多数报道的化合物 都是基于 $\mathrm{Mn}^{\mathrm{II}}$ 的高维磁有序化合物, 它们具有复杂的 结构和磁学行为, 且多个体系可以通过客体分子的得 失进行磁性质的调控. 通过选用多齿螯合配体，研究 者不仅实现了多个基于 $\left[\mathrm{Mo}(\mathrm{CN})_{7}\right]^{4-}$ 的低维分子磁性 材料的构筑，而且获得了高能垒单分子磁体、高能垒 单链磁体等的合成. 这些结果不仅证实了早期的理论 预测的结果，还为新的理论研究提供了实验模型. 然 而，三价Mo中心对光和空气敏感的特点使得基于 $\left[\mathrm{Mo}(\mathrm{CN})_{7}\right]^{4-}$ 的化合物相对于其他氰基金属而言依然 非常稀少, 这方面的工作还有待进一步研究. 值得指出 的是, 尽管 $\left[\mathrm{Mo}(\mathrm{CN})_{7}\right]^{4-}$ 离子在溶液中对空气非常敏感, 但基于 $\left[\mathrm{Mo}(\mathrm{CN})_{7}\right]^{4-}$ 的配合物的空气稳定性相对于 $\left[\mathrm{Mo}(\mathrm{CN})_{7}\right]^{4-}$ 构筑块而言有了较大的提高. 有些配合物 在空气中非常稳定，甚至可以通过热处理在空气中实
现单晶到单晶的转变过程 ${ }^{[77]}$. 但也有些配合物依然对 氧气较为敏感. 这可能取决于晶体的堆积方式: 不同 的堆积方式影响了氧气和配合物晶体中 $\mathrm{Mo}^{\mathrm{III}}$ 中心的作 用情况.

在后续的研究中，一些可能的研究思路包括但不 限于以下三点：(1) 基于 $\left[\mathrm{Mo}(\mathrm{CN})_{7}\right]^{4-}$ 的单分子磁体和 单链磁体. 由于合成上的难度, 构筑基于 $\left[\mathrm{Mo}(\mathrm{CN})_{7}\right]^{4-}$ 的低维分子纳米磁体仍是一项十分有挑战性的工作, 所报道的例子依然非常有限. 理论研究表明, $\mathrm{V}^{\mathrm{II}}$ 中心 可以和 $\mathrm{Mo}^{\mathrm{III}}$ 之间具有非常强的各向异性磁交换, 这对 于构筑高能垒分子纳米磁体非常有效. 然而, 由于低 价态 $\mathrm{V}^{\mathrm{II}}$ 离子对空气和水也极为敏感, 相应的 $\mathrm{V}^{\mathrm{II}}-\mathrm{Mo}^{\mathrm{III}}$ 单分子磁体和单链磁体也一直未见报道. (2) 其他金 属中心的 $\mathrm{M}-\mathrm{Mo}^{\mathrm{III}}$ 体系的构筑. 目前, 绝大多数基于 $\left[\mathrm{Mo}(\mathrm{CN})_{7}\right]^{4-}$ 的化合物都是基于 $\mathrm{Mn}^{\mathrm{II}}$ 的化合物, 基于其 他3 $\mathrm{d}$ 金属(如 $\mathrm{V}^{\mathrm{II}}, \mathrm{Co}^{\mathrm{II}}, \mathrm{Fe}^{\mathrm{II}}, \mathrm{Ni}^{\mathrm{II}}$ 等)化合物非常少见. 而 且, 由于反应的困难以及 $\mathrm{N}$ 原子和 $4 \mathrm{f}$ 金属相对较弱的配 位能力，至今还没有其和 $4 \mathrm{~d} 、 5 \mathrm{~d}$ 及 $4 \mathrm{f}$ 金属离子的金属 配合物的报道. 通过合成这些新的金属离子和 $\left[\mathrm{Mo}(\mathrm{CN})_{7}\right]^{4-}$ 所构筑的分子磁性材料, 不仅能够获得新 的结构类型, 而且可以研究新的磁相互作用及新的磁 学行为. (3) 基于 $\left[\mathrm{Mo}(\mathrm{CN})_{7}\right]^{4-}$ 的多功能分子材料. 分子 磁性材料的优势在于其功能易于复合调控. 通过引入 新的抗衡阳离子、新的功能化鳌合端基配体、新的功 能桥连配体等手段, 可能可以在基于 $\left[\mathrm{Mo}(\mathrm{CN})_{7}\right]^{4-}$ 的分 子磁性材料中引入其他功能性质, 如自旋交叉、光致 变色、介电和导电等, 构筑更为有趣的多功能分子磁 性材料.

总之, $\left[\mathrm{Mo}(\mathrm{CN})_{7}\right]^{4-}$ 构筑块在分子磁性材料中有着 独特的性质, 相关研究不仅对于拓展 $\left[\mathrm{Mo}(\mathrm{CN})_{7}\right]^{4-}$ 分子 磁性材料有重要意义, 也为构筑高能垒和高阻塞温度 的分子纳米磁体提供了新的途径. 我们相信, 基于此 构筑块的研究一定会产生更多具有新颖结构和性质的 分子磁性材料.

\section{参考文献}

1 Carlin RL, Van-Duyneveldt AJ. Magnetic Properties of Transition Metal Compounds. New York: Springer-Verlag, 1977

2 Carlin RL. Magnetochemistry. Berlin, Heidelberg: Springer-Verlag, 1986

3 Kahn O. Molecular Magnetism. New York: Wiley VCH, 1993

4 Miller JS, Drillon M. Magnetism: Molecules to Materials I-V. Weinheim: Wiley-VCH, 2002 
5 Herren F, Fischer P, Ludi A, Haelg W. Inorg Chem, 1980, 19: 956-959

6 Mallah T, Thiebaut S, Verdaguer M, Veillet P. Science, 1993, 262: 1554-1557

7 Entley WR, Girolami GS. Science, 1995, 268: 397-400

8 Ferlay S, Mallah T, Ouahès R, Veillet $\mathrm{P}$, Verdaguer M. Nature, 1995, 378: 701-703

9 Hatlevik Ø, Buschmann WE, Zhang J, Manson JL, Miller JS. Adv Mater, 1999, 11: 914-918

10 Sato O, Iyoda T, Fujishima A, Hashimoto K. Science, 1996, 272: 704-705

11 Sato O, Einaga Y, Fujishima A, Hashimoto K. Inorg Chem, 1999, 38: 4405-4412

12 Bleuzen A, Lomenech C, Escax V, Villain F, Varret F, Cartier dit Moulin C, Verdaguer M. J Am Chem Soc, 2000, 122: 6648-6652

13 Ruiz E, Rodríguez-Fortea A, Alvarez S, Verdaguer M. Chem-A Eur J, 2005, 11: 2135-2144

14 Visinescu D, Desplanches C, Imaz I, Bahers V, Pradhan R, Villamena FA, Guionneau P, Sutter JP. J Am Chem Soc, 2006, 128: 10202-10212

15 Wang XY, Avendaño C, Dunbar KR. Chem Soc Rev, 2011, 40: 3213-3238

16 Sieklucka B, Podgajny R, Korzeniak T, Przychodzeń P, Kania R. Comptes Rendus Chimie, 2002, 5: 639-649

17 Sieklucka B, Podgajny R, Przychodzeń P, Korzeniak T. Coord Chem Rev, 2005, 249: 2203-2221

18 Sieklucka B, Podgajny R, Pinkowicz D, Nowicka B, Korzeniak T, Bałanda M, Wasiutyński T, Pełka R, Makarewicz M, Czapla M, Rams M, Gawel B, Łasocha W. CrystEngComm, 2009, 11: 2032-2039, and references therein 2011, 50: 3973-3977 896-900

Song Y, Zhang P, Ren XM, Shen XF, Li YZ, You XZ. J Am Chem Soc, 2005, 127: 3708-3709

Chorazy S, Rams M, Hoczek A, Czarnecki B, Sieklucka B, Ohkoshi S, Podgajny R. Chem Commun, 2016, 52: 4772-4775

Venkatakrishnan TS, Sahoo S, Brefuel N, Duhayon C, Paulsen C, Barra AL, Ramasesha S, Sutter JP. J Am Chem Soc, 2010, 132: 6047-6056 Zhang YZ, Dolinar BS, Liu S, Brown AJ, Zhang X, Wang ZX, Dunbar KR. Chem Sci, 2018, 9: 119-124

Wei RM, Cao F, Li J, Yang L, Han Y, Zhang XL, Zhang Z, Wang XY, Song Y. Sci Rep, 2016, 6: 24372

Ohkoshi SI, Imoto K, Tsunobuchi Y, Takano S, Tokoro H. Nat Chem, 2011, 3: 564-569

Ohkoshi SI, Takano S, Imoto K, Yoshikiyo M, Namai A, Tokoro H. Nat Photon, 2014, 8: 65-71

Chorazy S, Podgajny R, Nakabayashi K, Stanek J, Rams M, Sieklucka B, Ohkoshi S. Angew Chem Int Ed, 2015, 54: 5093-5097

Podgajny R, Chorazy S, Nitek W, Rams M, Majcher AM, Marszałek B, Żukrowski J, Kapusta C, Sieklucka B. Angew Chem Int Ed, 2013, 52:

Chorazy S, Stanek JJ, Nogaś W, Majcher AM, Rams M, Kozieł M, Juszyńska-Gałązka E, Nakabayashi K, Ohkoshi S, Sieklucka B, Podgajny R. J Am Chem Soc, 2016, 138: 1635-1646

Chorazy S, Nakabayashi K, Imoto K, Mlynarski J, Sieklucka B, Ohkoshi S. J Am Chem Soc, 2012, 134: 16151-16154

Chorazy S, Podgajny R, Nitek W, Fic T, Görlich E, Rams M, Sieklucka B. Chem Commun, 2013, 49: 6731-6733

Magott M, Stefańczyk O, Sieklucka B, Pinkowicz D. Angew Chem Int Ed, 2017, 56: 13283-13287

Chibotaru LF, Hendrickx MFA, Clima S, Larionova J, Ceulemans A. J Phys Chem A, 2005, 109: 7251-7257

Mironov VS, Chibotaru LF, Ceulemans A. J Am Chem Soc, 2003, 125: 9750-9760

Young RC. J Am Chem Soc, 1932, 54: 1402-1405

Rossman GR, Tsay FD, Gray HB. Inorg Chem, 1973, 12: 824-829

Hursthouse MB, Malik KMA, Soares AM, Gibson JF, Griffith WP. Inorg Chim Acta, 1980, 45: L81-L82

Larionova J, Clérac R, Sanchiz J, Kahn O, Golhen S, Ouahab L. J Am Chem Soc, 1998, 120: 13088-13095

Larionova J, Sanchiz J, Kahn O, Gohlen S, Ouahab L. Chem Commun, 1998, 953-954

Larionova J, Kahn O, Golhen S, Ouahab L, Clérac R. Inorg Chem, 1999, 38: 3621-3627

Larionova J, Kahn O, Bartolome J, Burriel R, Castro M, Ksenofontov V, Gütlich P. Chem Mater, 1999, 11: 3400-3405

Larionova J, Willemin S, Donnadieu B, Henner B, Guerin C, Gillon B, Goujon A. J Phys Chem Solids, 2004, 65: 677-691

Larionova J, Kahn O, Gohlen S, Ouahab L, Clérac R. J Am Chem Soc, 1999, 121: 3349-3356

Kahn O, Larionova J, Ouahab L. Chem Commun, 1999, 945-952

Larionova J, Clérac R, Donnadieu B, Guérin C. Chem Eur J, 2002, 8: 2712-2716 

2015, 54: 9861-9865

Le Goff XF, Willemin S, Coulon C, Larionova J, Donnadieu B, Clérac R. Inorg Chem, 2004, 43: 4784-4786

Willemin S, Larionova J, Bolvin H, Donnadieu B, Le Goff XF, Ruiz E, Guérin C, Henner B. Polyhedron, 2005, 24: $1033-1046$

Kaur Sra A, Andruh M, Kahn O, Golhen S, Ouahab L, Yakhmi JV. Angew Chem Int Ed, 1999, 38: 2606-2609

Sra AK, Lahitite F, Yakhmi JV, Kahn O. Phys B-Condensed Matter, 2002, 321: 87-90

Tanase S, Tuna F, Guionneau P, Maris T, Rombaut G, Mathonière C, Andruh M, Kahn O, Sutter JP. Inorg Chem, 2003, 42: 1625-1631

Milon J, Daniel MC, Kaiba A, Guionneau P, Brandès S, Sutter JP. J Am Chem Soc, 2007, 129: 13872-13878

Tomono K, Tsunobuchi Y, Nakabayashi K, Ohkoshi S. Inorg Chem, 2010, 49: 1298-1300

Wang QL, Southerland H, Li JR, Prosvirin AV, Zhao H, Dunbar KR. Angew Chem Int Ed, 2012, 51: 9321-9324

Wang QL, Zhang YZ, Southerland H, Prosvirin AV, Zhao H, Dunbar KR. Dalton Trans, 2014, 43: 6802-6810

Wei XQ, Pi Q, Shen FX, Shao D, Wei HY, Wang XY. Dalton Trans, 2018, 47: 11873-11881

Shi L, Shao D, Shen F-, Wei X, Wang X. Chin J Chem, 2019, 37: 19-24

Wei XQ, Wang KJ, Wu DQ, Shao D, Shi L, Shen FX, Wei HY, Wang XY. Dalton Trans, 2019, 48: 8843-8852

Magott M, Dunbar KR, Pinkowicz D. Dalton Trans, 2019, 48: 15493-15500

Sra AK, Rombaut G, Lahitête F, Golhen S, Ouahab L, Mathonière C, Yakhmi JV, Kahn O. New J Chem, 2000, 24: 871-876

Wu DQ, Kempe D, Zhou Y, Deng LD, Shao D, Wei XQ, Shi L, Dunbar KR, Wang XY. Inorg Chem, 2017, 56: 7182-7189

Tomono K, Tsunobuchi Y, Nakabayashi K, Kosaka W, Matsuda T, Ohkoshi S. Chem Lett, 2009, 38: 810-811

Wang XY, Prosvirin A, Dunbar K. Angew Chem Int Ed, 2010, 49: 5081-5084

Kempe DK, Dolinar BS, Vignesh KR, Woods TJ, Saber MR, Dunbar KR. Chem Commun, 2019, 55: 2098-2101

Pradhan R, Desplanches C, Guionneau P, Sutter JP. Inorg Chem, 2003, 42: 6607-6609

Qian K, Huang XC, Zhou C, You XZ, Wang XY, Dunbar KR. J Am Chem Soc, 2013, 135: 13302-13305

Mironov VS. Inorg Chem, 2015, 54: 11339-11355

Wei XQ, Qian K, Wei HY, Wang XY. Inorg Chem, 2016, 55: 5107-5109

Wang K, Xia B, Wang QL, Ma Y, Liao DZ, Tang J. Dalton Trans, 2017, 46: 1042-1046

Shi L, Shao D, Wei X, Dunbar KR, Wang X. Angew Chem Int Ed, 2020, 59: 10379-10384

Sato O. Nat Chem, 2016, 8: 644-656

Liu W, Peng YY, Wu SG, Chen YC, Hoque MN, Ni ZP, Chen XM, Tong ML. Angew Chem Int Ed, 2017, 56: 14982-14986

Zeng MH, Yin Z, Tan YX, Zhang WX, He YP, Kurmoo M. J Am Chem Soc, 2014, 136: 4680-4688

Zhang X, Vieru V, Feng X, Liu JL, Zhang Z, Na B, Shi W, Wang BW, Powell AK, Chibotaru LF, Gao S, Cheng P, Long JR. Angew Chem Int Ed,

Zhang YJ, Liu T, Kanegawa S, Sato O. J Am Chem Soc, 2009, 131: 7942-7943

Milon J, Guionneau P, Duhayon C, Sutter JP. New J Chem, 2011, 35: 1211-1218

Wu DQ, Shao D, Wei XQ, Shen FX, Shi L, Kempe D, Zhang YZ, Dunbar KR, Wang XY. J Am Chem Soc, 2017, 139: 11714-11717 


\title{
Research progress in molecular magnetic materials based on the $\left[\mathrm{Mo}(\mathrm{CN})_{7}\right]^{4-}$ unit
}

\author{
Le Shi ${ }^{1}$, Dongqing $\mathrm{Wu}^{2}$, Xiaoqin $\mathrm{Wei}^{3}$, Xinyi Wang ${ }^{1 *}$ \\ ${ }^{1}$ State Key Laboratory of Coordination Chemistry, Collaborative Innovation Center of Advanced Microstructures, School of Chemistry and Chemical \\ Engineering, Nanjing University, Nanjing 210023, China \\ ${ }^{2}$ School of Chemistry and Chemical Engineering, Shangqiu Normal University, Shangqiu 476000, China \\ ${ }^{3}$ School of Chemistry and Chemical Engineering, Jinzhong University, Jinzhong 030619, China \\ *Corresponding author (email: wangxy66@nju.edu.cn)
}

\begin{abstract}
Molecular magnetic materials, assembled by spin carriers and organic ligands, are a class of compounds with specific magnetic properties. The $\left[\mathrm{Mo}(\mathrm{CN})_{7}\right]^{4-}$ building block with a trivalent $\mathrm{Mo}{ }^{\mathrm{III}}$ center has unique significance in this area. On one hand, strong magnetic coupling between the $\mathrm{Mo}^{\mathrm{III}}$ spin and other metal ions through the bridging cyanide groups is promising for the preparation of high $T_{\mathrm{c}}$ magnets. On the other hand, the strong magnetic anisotropy, arising from both the single-ion magnetic anisotropy of the $\mathrm{Mo}^{\mathrm{III}}$ center and also the strong anisotropic exchange interaction between other metal centers, provides new ideas for the construction of molecular nanomagnets. In this review, we summarized the research progress in the $\left[\mathrm{Mo}(\mathrm{CN})_{7}\right]^{4-}$-based molecular magnetic materials, including high-dimensional magnets, low-dimensional magnets and guest-switchable magnetic materials. Prospects of future studies in this specific field are also proposed.
\end{abstract}

Keywords: molecular magnetic materials, $\left[\mathrm{Mo}(\mathrm{CN})_{7}\right]^{4-}$, molecular-based magnets, anisotropic magnetic exchange, molecular nanomagnets

doi: $10.1360 /$ SSC-2020-0122 